\title{
Revisiting the generalized polar decomposition of Mueller matrices
}

\author{
Christian Heinrich ${ }^{1, *}$, Jean Rehbinder ${ }^{1}$, and Jihad Zallat $^{1}$ \\ ${ }^{1}$ ICube, Université de Strasbourg, CNRS (UMR 7357), 300 bld. S. Brant, CS 10413, 67412 Illkirch cedex, France \\ *Corresponding author: christian.heinrich@unistra.fr \\ Compiled July 7, 2020
}

\begin{abstract}
Mueller polarimetry is a powerful imaging modality which has been successfully applied to various application fields. Decomposition of Mueller matrices in elementary components is classically considered in order to unfold complex physical phenomena taking place in probed samples or scenes. In this context, the generalized polar decomposition, also known as the Lu and Chipman decomposition, is playing a prominent role. In this article, we show that the set of candidate generalized polar decompositions is richer than the set used so far. Negative-determinant Mueller matrices are naturally addressed in the proposed framework. We show that taking into account those supplementary polar decompositions addresses issues raised in the literature. Application is carried out on synthetic and on measured Mueller matrices.
\end{abstract}

(C) 2020 Optical Society of America

http://dx.doi.org/10.1364/ao.XX.XXXXXX

\section{INTRODUCTION}

Mueller polarimetry is a powerful imaging modality which has successfully met many application fields such as medicine, biology, engineering, and material science (see e.g., [1-7]). This probing technique gives access into the structure of the sample of interest. Information is held by a Mueller matrix, which is a $4 \times 4$ real matrix modeling the relation between an incoming Stokes vector and the corresponding outcoming Stokes vector. Because of its intrinsic advantages, the Stokes-Mueller formalism is one of the most used in polarimetry. It is in fact capable of representing any state of polarization by a Stokes vector while the Mueller matrix is capable of accounting for any possible linear interaction between a medium and the incident light that provides the outgoing Stokes vector. Thus, for a given geometric configuration, the Mueller matrix contains all the energetic and polarimetric information related to the medium.

The interpretation of Mueller matrices has been and still is of wide scientific interest, with a view to unfolding and interpreting complex physical phenomena taking place in structured media. In this context, the generalized polar decomposition (GPD), also known as the Lu and Chipman decomposition [8] (see also $[9,10]$ ) is playing a central role. Many other decompositions have been proposed (see e.g., [9-13]). In the GPD, a given Mueller matrix $\boldsymbol{M}$ is decomposed as a product $\boldsymbol{M}=\boldsymbol{M}_{\Delta} \boldsymbol{M}_{\mathrm{R}} \boldsymbol{M}_{\mathrm{D}}$, where $\boldsymbol{M}_{\Delta}, \boldsymbol{M}_{\mathrm{R}}$, and $\boldsymbol{M}_{\mathrm{D}}$ are Mueller matrices of a depolarizer, a retarder, and a diattenuator. Such a decomposition allows to decouple the different polarimetric effects. With this point of view, the medium of interest is modeled as a succession of three layers [10], which gives insight into the physical structure of interest. Besides, it may also be mentioned that the physical parameters of the set $\left\{\boldsymbol{M}_{\Delta}, \boldsymbol{M}_{\mathrm{R}}, \boldsymbol{M}_{\mathrm{D}}\right\}$ may constitute interesting features for machine learning algorithms, with a view to tissue classification and early diagnosis [14, 15].

Scientific interest has been devoted to the particular case of negative-determinant Mueller matrices (see e.g., [16] and the references therein; see e.g., [17] for the physical meaning of negative determinant Mueller matrices). In this case, a Mueller matrix is still decomposed as a product $\boldsymbol{M}=\boldsymbol{M}_{\Delta} \boldsymbol{M}_{\mathrm{R}} \boldsymbol{M}_{\mathrm{D}}$, but the decomposition is different from the $\mathrm{Lu}$ and Chipman one.

In the original work [8], the choice between different potential decompositions is achieved by minimizing the retardance. In this article, minimizing the retardance is merely a way to address identifiability issues; the authors do not claim that this approach yields an estimation closer to the ground truth than a concurrent approach. We may question whether minimizing the retardance is the best choice to be made from a physical point of view. For example, in [section 3, 16], the authors make a choice with respect to the orientation of the rotation axis, and this may be in contradiction with the minimization of the retardance. Another approach, also to be found in [section 3,16], is to employ continuity arguments on the depolarization image. This would certainly be an interesting point of view for the estimation of a GPD. Finally, in the present work, indetermination is always 
solved by minimizing the retardance, to be consistent with [8]. Considering other criteria, such as the rotation axis orientation or as continuity considerations, is left for future work.

The contribution of this article resides in showing that the original GPD stems from a subset of the admissible candidates. Considering the entire set of GPD candidates may change the GPD retained, since minimization of the retardance is achieved on a richer set of candidates. In the case of negative-determinant Mueller matrices, the solution of [16] appears naturally as a candidate. Nevertheless, for some negative-determinant Mueller matrices, the proposed approach also reaches a lower retardance than the one in [16].

In this work, we focus on decomposition, optimization, and estimation issues. We leave physical interpretations aside, this aspect being widely covered in the literature (see e.g., [8-10]).

The article is structured as follows. In the second section, we recall the GPD and define the variables at stake. In the third section, we revisit the GPD construction and we derive the complete set of GPD candidates attached to a given Mueller matrix. Simulations using random synthetic Mueller matrices are proposed to supplement the derivations. In the fourth section, we apply the proposed decomposition methodology to a biological sample. Conclusion is drawn in the last section.

\section{THE GENERALIZED POLAR DECOMPOSITION}

The goal of this section is to recall the GPD of a Mueller matrix, as taken from [8]. All notations are standard and stem from the literature.

A Mueller matrix $\boldsymbol{M}$ can be written as $\boldsymbol{M}=m_{00}\left[\begin{array}{ll}1 & \boldsymbol{D}^{\mathrm{t}} \\ \boldsymbol{P} & \boldsymbol{m}\end{array}\right]$ $[8,9,18]$, and is decomposed as $\boldsymbol{M}=\boldsymbol{M}_{\Delta} \boldsymbol{M}_{\mathrm{R}} \boldsymbol{M}_{\mathrm{D}}$, where $\boldsymbol{M}_{\Delta}$, $\boldsymbol{M}_{\mathrm{R}}$, and $\boldsymbol{M}_{\mathrm{D}}$ represent respectively a depolarizer, a retarder, and a diattenuator. The matrices at stake write

$\boldsymbol{M}_{\Delta}=\left[\begin{array}{cc}1 & \boldsymbol{0}^{\mathrm{t}} \\ \boldsymbol{P}_{\Delta} & \boldsymbol{m}_{\Delta}\end{array}\right], \quad \boldsymbol{M}_{\mathrm{R}}=\left[\begin{array}{cc}1 & \boldsymbol{0}^{\mathrm{t}} \\ \mathbf{0} & \boldsymbol{m}_{\mathrm{R}}\end{array}\right], \quad \boldsymbol{M}_{\mathrm{D}}=m_{00}\left[\begin{array}{cc}1 & \boldsymbol{D}^{\mathrm{t}} \\ \boldsymbol{D} & \boldsymbol{m}_{\mathrm{D}}\end{array}\right]$.

The diattenuator vector $\boldsymbol{D}$ has norm $D(0 \leqslant D \leqslant 1)$. The polarizance vector $\boldsymbol{P}$ has norm $P(0 \leqslant P \leqslant 1)$. We define the unit vectors $\widehat{\boldsymbol{D}}$ and $\widehat{\boldsymbol{P}}$ along $\boldsymbol{D}$ and $\boldsymbol{P}: \boldsymbol{D}=D \widehat{\boldsymbol{D}}, \boldsymbol{P}=P \widehat{\boldsymbol{P}}$. We have:

$$
m_{\mathrm{D}}=\sqrt{1-D^{2}} \mathbf{I}+\left(1-\sqrt{1-D^{2}}\right) \widehat{\mathbf{D}} \widehat{\mathbf{D}}^{\mathrm{t}}
$$

Matrix $\boldsymbol{M}_{\mathrm{D}}$ can therefore be calculated from $m_{00}$ and from the diattenuator vector $\boldsymbol{D}$. Besides, the equations given above yield:

$$
\left\{\begin{array}{l}
\boldsymbol{P}=\boldsymbol{P}_{\Delta}+m_{\Delta} m_{\mathrm{R}} \boldsymbol{D} \\
\boldsymbol{m}=\boldsymbol{P}_{\Delta} D^{\mathrm{t}}+m_{\Delta} m_{\mathrm{R}} m_{\mathrm{D}}
\end{array}\right.
$$

From $m_{\mathrm{D}} \boldsymbol{D}=\boldsymbol{D}$, we get

$$
\begin{aligned}
\boldsymbol{P} & =\boldsymbol{P}_{\Delta}+\boldsymbol{m}_{\Delta} \boldsymbol{m}_{\mathrm{R}} \boldsymbol{m}_{\mathrm{D}} \boldsymbol{D}=\boldsymbol{P}_{\Delta}+\left(\boldsymbol{m}-\boldsymbol{P}_{\Delta} \boldsymbol{D}^{\mathrm{t}}\right) \boldsymbol{D} \\
& =\left(1-D^{2}\right) \boldsymbol{P}_{\Delta}+\boldsymbol{m} \boldsymbol{D} .
\end{aligned}
$$

By definition, submatrices $m_{R}$ and $m_{\Delta}$ are respectively a 3D rotation matrix and a symmetric matrix [8].

It should be noticed that $\boldsymbol{M}_{\Delta}$ is not a physically admissible Mueller matrix (see e.g., [section 5.12.1, page 39, 9] ; see [section 5.4,9] for the characterization of Mueller matrices). More precisely, Lu and Chipman decompose $\boldsymbol{M}$ as

$$
\boldsymbol{M}=\boldsymbol{M}_{\Delta} \boldsymbol{M}_{\mathrm{R}} \boldsymbol{M}_{\mathrm{D}}=\boldsymbol{M}_{\Delta} \boldsymbol{M}_{\mathrm{R}}\left[m_{00}\left[\begin{array}{cc}
1 & \boldsymbol{D}^{\mathrm{t}} \\
\boldsymbol{D} & \boldsymbol{m}_{\mathrm{D}}
\end{array}\right]\right] .
$$

Gil [9] decomposes $\boldsymbol{M}$ as

$$
\boldsymbol{M}=\left[\begin{array}{ll}
c & \boldsymbol{M}_{\Delta}
\end{array}\right]\left[b \boldsymbol{M}_{\mathrm{R}}\right]\left[a\left[\begin{array}{cc}
1 & \boldsymbol{D}^{\mathrm{t}} \\
\boldsymbol{D} & \boldsymbol{m}_{\mathrm{D}}
\end{array}\right]\right],
$$

with the physical admissibility conditions $a b c=m_{00}, a, b, c \geqslant 0$, $a(1+D) \leqslant 1, b \leqslant 1, c\left(1+\left\|\boldsymbol{P}_{\Delta}\right\|\right) \leqslant 1$. In the framework of Gil, Lu and Chipman choose $a=m_{00}, b=1, c=1$. With $c=1$, condition $c\left(1+\left\|\boldsymbol{P}_{\Delta}\right\|\right) \leqslant 1$ cannot be met, unless $\left\|\boldsymbol{P}_{\Delta}\right\|=0$. This point may be addressed by post processing the Lu and Chipman decomposition, by distributing $m_{00}$ on $a, b$, and $c$, without any further impact on the decomposition.

We define matrix $\boldsymbol{M}^{\prime}$ as $\boldsymbol{M}^{\prime}=\boldsymbol{M}_{\Delta} \boldsymbol{M}_{\mathrm{R}}$ [Eq. 48, 8]. Thus we get $\boldsymbol{M}^{\prime}=\left[\begin{array}{cc}1 & \boldsymbol{0}^{\mathrm{t}} \\ \boldsymbol{P}_{\Delta} & \boldsymbol{m}^{\prime}\end{array}\right]$, where $\boldsymbol{m}^{\prime}=\boldsymbol{m}_{\Delta} \boldsymbol{m}_{\mathrm{R}}$.

Let us define vectors $v_{\mathrm{D} 1}, \boldsymbol{v}_{\mathrm{D} 2}, \boldsymbol{v}_{\mathrm{D} 3}, \boldsymbol{v}_{\mathrm{D} 4}$ respectively as

$$
\frac{1}{\sqrt{2}}\left[\begin{array}{c}
1 \\
\boldsymbol{D}
\end{array}\right], \quad\left[\begin{array}{c}
0 \\
\widehat{D}_{\perp 1}
\end{array}\right], \quad\left[\begin{array}{c}
0 \\
\widehat{D}_{\perp 2}
\end{array}\right], \quad \frac{1}{\sqrt{2}}\left[\begin{array}{c}
1 \\
-\widehat{D}
\end{array}\right],
$$

where $\widehat{\boldsymbol{D}}_{\perp 1}$ and $\widehat{\boldsymbol{D}}_{\perp 2}$ are two orthonormal vectors, orthogonal to $\widehat{D}$. Those four vectors are unit-normed and are eigenvectors of $\boldsymbol{M}_{\mathrm{D}}$, with eigenvalues

$$
S_{\mathrm{D}}=m_{00}\left[1+D ; \sqrt{1-D^{2}} ; \sqrt{1-D^{2}} ; 1-D\right],
$$

appearing in decreasing order (see [Eqs. $20 \& 21,8]$ ). There-

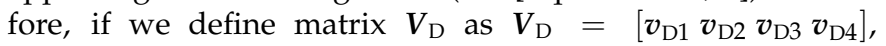
$\left(V_{\mathrm{D}}, \operatorname{diag}\left(S_{\mathrm{D}}\right), V_{\mathrm{D}}\right)$ is a singular value decomposition (SVD) of $\boldsymbol{M}_{\mathrm{D}}: \boldsymbol{M}_{\mathrm{D}}=\boldsymbol{V}_{\mathrm{D}} \operatorname{diag}\left(S_{\mathrm{D}}\right) \boldsymbol{V}_{\mathrm{D}}^{t}$. As a consequence, $\boldsymbol{M}_{\mathrm{D}}$ is invertible if and only if $D<1$.

Two cases are to be distinguished: the $\boldsymbol{M}_{\mathrm{D}}$ invertible case, and the $\boldsymbol{M}_{\mathrm{D}}$ singular case.

\section{A. The $M_{\mathrm{D}}$ invertible case}

If $\boldsymbol{M}_{\mathrm{D}}$ is invertible, $\boldsymbol{M}^{\prime}$ is uniquely defined as $\boldsymbol{M}^{\prime}=\boldsymbol{M} \boldsymbol{M}_{\mathrm{D}}^{-1}$, and we get $\boldsymbol{P}_{\Delta}$ from

$$
\boldsymbol{P}_{\Delta}=\frac{1}{1-D^{2}}(\boldsymbol{P}-\boldsymbol{m} \boldsymbol{D}) \text {. }
$$

Let $(\boldsymbol{V}, \boldsymbol{S}, \boldsymbol{U})$ be a SVD of $\boldsymbol{m}^{\prime}$, i.e., $\boldsymbol{m}^{\prime}=\boldsymbol{V} \boldsymbol{S} \boldsymbol{U}^{\mathrm{t}}$. If $\boldsymbol{m}^{\prime}$ is invertible, $m_{\Delta}$ is determined using [Eq. 52, 8], or equivalently using [Eq. B2, 8] as

$$
m_{\Delta}= \pm V S V^{\mathrm{t}}
$$

where the + sign is used if det $\boldsymbol{m}^{\prime}>0$, whereas the - sign is used if det $m^{\prime}<0$. See also [Eq. 230, 9].

Finally, $m_{\mathrm{R}}=m_{\Delta}^{-1} m^{\prime}$ and the Lu and Chipman GPD (LCGPD) is complete. Equivalently, we may use

$$
m_{\mathrm{R}}= \pm V U^{\mathrm{t}}
$$

with the same rule as above for choosing the sign.

If $\boldsymbol{m}^{\prime}$ is singular, $\boldsymbol{m}_{\Delta}$ is singular as well and $\boldsymbol{m}_{\mathrm{R}}$ is not unique. This case is addressed in [Appendix B, 8]. We do not detail this case here, since this will be one of the topics of the next section.

\section{B. The $M_{\mathrm{D}}$ singular case}

If $M_{\mathrm{D}}$ is singular (i.e., if $D=1$ and $\boldsymbol{D}=\widehat{D}$ ), then matrix $\boldsymbol{M}$ writes $\boldsymbol{M}=m_{00}\left[\begin{array}{cc}1 & \boldsymbol{D}^{\mathrm{t}} \\ \boldsymbol{P} & \boldsymbol{P} \boldsymbol{D}^{\mathrm{t}}\end{array}\right]$ (see section $\mathrm{A}$ in the appendix). The LC-GDP writes [Eq. A3, 8]

$$
\boldsymbol{M}_{\Delta}=\left[\begin{array}{cc}
1 & \mathbf{0}^{\mathrm{t}} \\
\mathbf{0} & P_{\mathbf{I}}
\end{array}\right], \quad \boldsymbol{M}_{\mathrm{R}}=\left[\begin{array}{cc}
1 & \mathbf{0}^{\mathrm{t}} \\
\mathbf{0} & \mathcal{R}(\boldsymbol{D}, \widehat{\boldsymbol{P}})
\end{array}\right]
$$


where $\mathcal{R}\left(\boldsymbol{u}_{1}, \boldsymbol{u}_{2}\right)$ is the minimal angle rotation matrix that transforms unit-length vector $\boldsymbol{u}_{1}$ to unit-length vector $\boldsymbol{u}_{2}\left(\boldsymbol{u}_{1}\right.$ and $\boldsymbol{u}_{2}$ are supposed linearly independent). This is a rotation around $\boldsymbol{u}_{1} \times \boldsymbol{u}_{2}$ normalized to unit-length, and the rotation angle $\theta$ is the angle formed by $\boldsymbol{u}_{1}$ and $\boldsymbol{u}_{2}$ (see e.g., [19]). Let $\boldsymbol{u}=\frac{1}{\left\|\boldsymbol{u}_{1} \times \boldsymbol{u}_{2}\right\|} \boldsymbol{u}_{1} \times \boldsymbol{u}_{2}=\left[\begin{array}{lll}u_{x} & u_{y} & u_{z}\end{array}\right]^{t}$. If we define the skewsymmetric matrix $[\boldsymbol{u}]_{\mathrm{X}}$ as

$$
[\boldsymbol{u}]_{\mathrm{X}}=\left[\begin{array}{ccc}
0 & -u_{z} & u_{y} \\
u_{z} & 0 & -u_{x} \\
-u_{y} & u_{x} & 0
\end{array}\right]
$$

this matrix being such that $[u]_{\mathrm{X}} \boldsymbol{w}=\boldsymbol{u} \times \boldsymbol{w}$ for any vector $w$, we have

$$
\mathcal{R}\left(\boldsymbol{u}_{1}, \boldsymbol{u}_{2}\right)=\cos \theta \mathbf{I}+\sin \theta[\boldsymbol{u}]_{X}+(1-\cos \theta) \boldsymbol{u} \boldsymbol{u}^{\mathrm{t}},
$$

which may also be written (Rodrigues formula, see e.g., [19])

$$
\mathcal{R}\left(\boldsymbol{u}_{1}, \boldsymbol{u}_{2}\right)=\mathbf{I}+\sin \theta[\boldsymbol{u}]_{\mathrm{X}}+(1-\cos \theta)[\boldsymbol{u}]_{\mathrm{X}}^{2},
$$

where we used the relation $[\boldsymbol{u}]_{\mathrm{X}}^{2}=\boldsymbol{u} \boldsymbol{u}^{\mathrm{t}}-\mathbf{I}$. Changing the sign of $\boldsymbol{u}$ amounts to changing the sign of $\theta$.

In the appendix of this article, we will also use the notation $\mathcal{R}(\boldsymbol{u}, \theta)$ for the rotation of angle $\theta$ around vector $\boldsymbol{u}$.

In fact, $\boldsymbol{M}_{\mathrm{R}}$ in [8] is written $\left[\begin{array}{cc}1 & \mathbf{0}^{\mathrm{t}} \\ \mathbf{0} & \mathcal{R}(\widehat{\boldsymbol{P}}, \boldsymbol{D})\end{array}\right]$, which amounts to changing the sign of the rotation angle. But on the other hand, matrix $m_{\mathrm{R}}$ in [8] is the rotation of angle $-R$ [Eq. 15, 8]. Therefore, in [8] we have a double sign flip which cancels out.

\section{REVISITING THE GENERALIZED POLAR DECOMPO- SITION}

We aim at devising the set of all GPDs attached to a given Mueller matrix. One decomposition will have to be selected among all GPDs. As in [8], we will retain the one minimizing the retardance $R$, which is the rotation angle associated to $m_{R}$.

This section is divided in four parts. We first address the $\boldsymbol{M}_{\mathrm{D}}$ invertible case, we then deal with the $\boldsymbol{M}_{\mathrm{D}}$ singular case. In the third part, we focus on accounting for physical constraints. The fourth section proposes two case studies on synthetic Mueller matrices.

Definition: $\Delta \mathrm{RF} . \quad\left(m_{\Delta}, m_{\mathrm{R}}\right)$ is a $\Delta \mathrm{R}$ factorization $(\Delta \mathrm{RF})$ of a given matrix $m^{\prime}$ if

$$
m^{\prime}=m_{\Delta} m_{\mathrm{R}}, \quad m_{\mathrm{R}} m_{\mathrm{R}}^{\mathrm{t}}=\mathrm{I}, \quad \operatorname{det} m_{\mathrm{R}}=1, \quad m_{\Delta}=m_{\Delta}^{\mathrm{t}} .
$$

\section{A. The $M_{\mathrm{D}}$ invertible case}

In this case, $\boldsymbol{M}^{\prime}$ and $\boldsymbol{m}^{\prime}$ are uniquely defined. We aim at decomposing $\boldsymbol{m}^{\prime}$ as $\boldsymbol{m}^{\prime}=\boldsymbol{m}_{\Delta} \boldsymbol{m}_{\mathrm{R}}$. Let $\left(\boldsymbol{V}^{(p)}, \boldsymbol{S}, \boldsymbol{U}^{(p)}\right)$ be a particular SVD of $m^{\prime}$, such as given by a numerical software, or as chosen by the investigator. The singular values being unique (see e.g., [section 2, 20]), we drop $(p)$ and write $S$ instead of $\boldsymbol{S}^{(p)}$. Then $m^{\prime}$ admits at least one $\triangle R F$, which may be computed from the SVD [Eqs. B2 \& B3, 8]:

$$
\left\{\begin{array}{l}
\boldsymbol{m}_{\Delta}^{(p)}=e^{(p)} \boldsymbol{V}^{(p)} S \boldsymbol{V}^{(p) t} \\
\boldsymbol{m}_{\mathrm{R}}^{(p)}=e^{(p)} \boldsymbol{V}^{(p)} \boldsymbol{U}^{(p) t}, \\
e^{(p)}= \pm 1 .
\end{array}\right.
$$

The sign of $e^{(p)}$ is such that $\operatorname{det} m_{\mathrm{R}}^{(p)}=1$.

We aim at devising the set of all $\triangle \mathrm{RFs}$ attached to $\boldsymbol{m}^{\prime}$. Let $\left(\boldsymbol{m}_{\Delta}^{(g)}, \boldsymbol{m}_{\mathrm{R}}^{(g)}\right)$ be a general $\Delta \mathrm{RF}$ of $\boldsymbol{m}^{\prime}$ (i.e., any $\Delta \mathrm{RF}$ of $\boldsymbol{m}^{\prime}$ ). Matrix $\boldsymbol{m}_{\Delta}^{(g)}$ being real symmetric, it may be written $\boldsymbol{m}_{\Delta}^{(g)}=\boldsymbol{V}_{\Delta} \boldsymbol{S}_{\Delta} \boldsymbol{V}_{\Delta}^{\mathrm{t}}$ (spectral theorem for normal matrices, see e.g., [section 2.5, 21]) where we suppose, without loss of generality, that the diagonal matrix $S_{\Delta}$ is such that $\left|s_{\Delta 1}\right| \geqslant\left|s_{\Delta 2}\right| \geqslant\left|s_{\Delta 3}\right|$ (modification of the order of the $\left|s_{\Delta i}\right|$ 's is achieved by permutation of the columns of $\left.V_{\Delta}\right)$. Thereby

$$
\left\{\begin{array}{l}
\boldsymbol{m}^{\prime} \boldsymbol{m}^{\prime \mathrm{t}}=\boldsymbol{m}_{\Delta}^{(g)} \boldsymbol{m}_{\Delta}^{(g) t}, \\
\boldsymbol{m}^{\prime} \boldsymbol{m}^{\prime \mathrm{t}}=\boldsymbol{V}^{(p)} S^{2} \boldsymbol{V}^{(p) t} \\
\boldsymbol{m}_{\Delta}^{(g)} \boldsymbol{m}_{\Delta}^{(g) t}=\boldsymbol{V}_{\Delta} S_{\Delta}^{2} \boldsymbol{V}_{\Delta}^{\mathrm{t}} .
\end{array}\right.
$$

We notice that $\left(\boldsymbol{V}_{\Delta}, \boldsymbol{S}_{\Delta}^{2}, \boldsymbol{V}_{\Delta}\right)$ and $\left(\boldsymbol{V}^{(p)}, \boldsymbol{S}^{2}, \boldsymbol{V}^{(p)}\right)$ are two SVDs of the same matrix. As a consequence (see section $B$ in the appendix), $S_{\Delta}^{2}=S^{2}$ and there exists $Q_{1}$ such that $V_{\Delta}=V^{(p)} Q_{1}$ With $\varepsilon=\operatorname{diag}\left(\left[\begin{array}{lll} \pm 1 & \pm 1 & \pm 1\end{array}\right]\right)=\operatorname{diag}\left(\left[\begin{array}{lll}\varepsilon_{1} & \varepsilon_{2} & \varepsilon_{3}\end{array}\right]\right), S_{\Delta}=$ $\varepsilon S$. The structure of $Q_{1}$ is determined by the structure of the singular values $s_{1}, s_{2}, s_{3}$ (see the appendix, section B, Eq. 5, which reads $\left.\widehat{V}=V Q_{1}\right)$. Thus, for any $\boldsymbol{m}_{\Delta}^{(g)}$ and considering any given $\operatorname{SVD}\left(\boldsymbol{V}^{(p)}, \boldsymbol{S}, \boldsymbol{U}^{(p)}\right)$ of $\boldsymbol{m}^{\prime}$, there exists $\left(\boldsymbol{Q}_{1}, \boldsymbol{\varepsilon}\right)$ such that

$$
\boldsymbol{m}_{\Delta}^{(g)}=\boldsymbol{V}^{(p)} Q_{1} \varepsilon S Q_{1}^{\mathbf{t}} \boldsymbol{V}^{(p) t}
$$

The goal is to capture all $m_{\Delta}^{(g)}$ 's. This amounts to capturing all couples $\left(\boldsymbol{Q}_{1}, \boldsymbol{\varepsilon}\right)$ which, associated to the SVD $\left(\boldsymbol{V}^{(p)}, \boldsymbol{S}, \boldsymbol{U}^{(p)}\right)$, will yield a $\triangle \mathrm{RF}$. The symmetry condition on $m_{\Delta}^{(g)}$ being satisfied for any couple $\left(Q_{1}, \varepsilon\right)$, the stake is to find a matching $m_{\mathrm{R}}^{(g)}$. The ultimate goal being to capture all $\Delta \mathrm{RFs}$, we aim at capturing all triples $\left(Q_{1}, \varepsilon, m_{\mathrm{R}}^{(g)}\right)$ which, associated to the SVD $\left(\boldsymbol{V}^{(p)}, \boldsymbol{S}, \boldsymbol{U}^{(p)}\right)$, will yield a $\Delta \mathrm{RF}$.

Let us write a $\boldsymbol{m}_{\mathrm{R}}^{(g)}$ candidate as $\boldsymbol{m}_{\mathrm{R}}^{(g)}=\boldsymbol{V}^{(p)} \boldsymbol{Q}_{1} \boldsymbol{\varepsilon} \boldsymbol{U}_{\mathrm{R}}^{\mathrm{t}}$. Matrices $V^{(p)}, Q_{1}$, and $\varepsilon$ being invertible, any $3 \times 3$ real matrix may be written $\boldsymbol{V}^{(p)} \boldsymbol{Q}_{1} \varepsilon \boldsymbol{U}_{\mathrm{R}}^{\mathrm{t}}$, with a proper $\boldsymbol{U}_{\mathrm{R}}$. We aim at finding the necessary and sufficient conditions on $\left(Q_{1}, \varepsilon, U_{R}\right)$ such that $\left(Q_{1}, \varepsilon, U_{R}\right)$ will yield a $\Delta R F$ of $\boldsymbol{m}^{\prime}$.

The necessary conditions are

1. $\boldsymbol{m}_{\mathrm{R}}^{(g)} \boldsymbol{m}_{\mathrm{R}}^{(g) t}=\mathbf{I}\left(\Leftrightarrow \boldsymbol{m}_{\mathrm{R}}^{(g) t} \boldsymbol{m}_{\mathrm{R}}^{(g)}=\mathbf{I}\right)$, which yields $\boldsymbol{U}_{\mathrm{R}} \boldsymbol{U}_{\mathrm{R}}^{\mathrm{t}}=$ $U_{\mathrm{R}}^{\mathrm{t}} \boldsymbol{U}_{\mathrm{R}}=$ I. Matrix $\boldsymbol{U}_{\mathrm{R}}$ must be orthonormal;

2. $\boldsymbol{m}^{\prime}=\boldsymbol{m}_{\Delta}^{(g)} \boldsymbol{m}_{\mathrm{R}}^{(g)}=\left[\boldsymbol{V}^{(p)} \boldsymbol{Q}_{1} \varepsilon S \boldsymbol{Q}_{1}^{\mathrm{t}} \boldsymbol{V}^{(p) t}\right]\left[\boldsymbol{V}^{(p)} \boldsymbol{Q}_{1} \varepsilon \boldsymbol{U}_{\mathrm{R}}^{\mathrm{t}}\right]$. Thus $\boldsymbol{m}^{\prime}=\boldsymbol{V}^{(p)} \boldsymbol{Q}_{1} S \boldsymbol{U}_{\mathrm{R}}^{\mathrm{t}}$. On the other hand, we have $\boldsymbol{m}^{\prime}=\boldsymbol{V}^{(p)} S \boldsymbol{U}^{(p) t}$.

We now have two SVDs of $\boldsymbol{m}^{\prime}:\left(\boldsymbol{V}^{(p)} Q_{1}, S, \boldsymbol{U}_{\mathrm{R}}\right)$ and $\left(\boldsymbol{V}^{(p)}, \boldsymbol{S}, \boldsymbol{U}^{(p)}\right)$. Using section B in the appendix, we may state that there exists $Q_{2}$ such that $\boldsymbol{U}_{\mathrm{R}}=\boldsymbol{U}^{(p)} \boldsymbol{Q}_{2}$ (see Eq. 6, which reads $\widehat{W}=W Q_{2}$ ), and that there exists $Q_{3}$ such that $V^{(p)} Q_{1}=V^{(p)} Q_{3}$ (obviously, $Q_{3}$ must be equal to $Q_{1}$ ).

3. $\operatorname{det} \boldsymbol{m}_{\mathrm{R}}^{(g)}=1 \Rightarrow \operatorname{det}\left(\boldsymbol{V}^{(p)} \boldsymbol{Q}_{1} \varepsilon \boldsymbol{U}_{\mathrm{R}}^{\mathrm{t}}\right)=1$. 
Considering $\boldsymbol{U}_{\mathrm{R}}=\boldsymbol{U}^{(p)} \boldsymbol{Q}_{2}$, condition 1 above is satisfied, and condition 3 writes det $\left(\boldsymbol{V}^{(p)} \boldsymbol{Q}_{1} \varepsilon \boldsymbol{Q}_{2}^{\mathrm{t}} \boldsymbol{U}^{(p) t}\right)=1$. Those necessary conditions are sufficient: when met, they yield a $\triangle \mathrm{RF}$ of $m^{\prime}$.

Conclusion: provided that $\operatorname{det}\left(\boldsymbol{V}^{(p)} \boldsymbol{Q}_{1} \varepsilon \boldsymbol{Q}_{2}^{\mathrm{t}} \boldsymbol{U}^{(p) t}\right)=1$,

$$
\left(\boldsymbol{V}^{(p)} Q_{1} \varepsilon S Q_{1}^{\mathrm{t}} \boldsymbol{V}^{(p) t}, \boldsymbol{V}^{(p)} Q_{1} \varepsilon Q_{2}^{\mathrm{t}} \boldsymbol{U}^{(p) t}\right)
$$

is a $\triangle R F$ of $m^{\prime}$.

Given a particular SVD $\left(\boldsymbol{V}^{(p)}, \boldsymbol{S}, \boldsymbol{U}^{(p)}\right)$ of $\boldsymbol{m}^{\prime}$, we capture the entire set of $\Delta$ RFs by allowing $Q_{1}, Q_{2}$, and $\varepsilon$ to vary.

Structure. Eight different cases can be distinguished, according to the structure of the singular values $s_{1}, s_{2}, s_{3}$ (see also section D in the appendix)):

- $\operatorname{case}(\mathrm{a}): s_{1}>s_{2}>s_{3}>0\left(\Rightarrow Q_{1}=Q_{2}\right)$;

- case (b): $s_{1}=s_{2}>s_{3}>0\left(\Rightarrow Q_{1}=Q_{2}\right)$;

- $\operatorname{case}(\mathrm{c}): s_{1}>s_{2}=s_{3}>0\left(\Rightarrow Q_{1}=Q_{2}\right)$;

- case $(\mathrm{d}): s_{1}=s_{2}=s_{3}>0\left(\Rightarrow Q_{1}=Q_{2}\right)$;

- $\operatorname{case}(\mathrm{e}): s_{1}=s_{2}>s_{3}=0$;

- $\operatorname{case}(\mathrm{f}): s_{1}>s_{2}>s_{3}=0$;

- $\operatorname{case}(\mathrm{g}): s_{1}>s_{2}=s_{3}=0$;

- $\operatorname{case}(\mathrm{h}): s_{1}=s_{2}=s_{3}=0$.

Properties. Considering for each case (a)-(h) the particular structure of $Q_{1}, Q_{2}$, and $S$, we see that relations

$$
S Q_{2}=Q_{1} S, \quad S Q_{1}=Q_{1} S, \quad S Q_{2}=Q_{2} S
$$

hold.

Interpretation: linking the $\mathrm{Lu}$ and Chipman decomposition to the proposed decomposition. Let $\boldsymbol{R}_{p}=e^{(p)} \boldsymbol{V}^{(p)} \boldsymbol{Q}_{1} \varepsilon \boldsymbol{Q}_{2}^{t} \boldsymbol{V}^{(p) t}$. It may easily be checked that matrix $\boldsymbol{R}_{p}$ is a rotation matrix, thereby satisfying $\boldsymbol{R}_{p}^{t} \boldsymbol{R}_{p}=\mathbf{I}$.

Inserting the product $\boldsymbol{R}_{p}^{t} \boldsymbol{R}_{p}$ in the Lu and Chipman decomposition ( $\boldsymbol{m}_{\Delta-\mathrm{LC}}$ and $\boldsymbol{m}_{\mathrm{R}-\mathrm{LC}}$ are taken from Eqs. (2)), we get:

$$
m^{\prime}=\left[m_{\Delta-\mathrm{LC}} \boldsymbol{R}_{p}^{t}\right]\left[\boldsymbol{R}_{p} m_{\mathrm{R}-\mathrm{LC}}\right] .
$$

Using the first equation in the Properties above, it can easily be checked that the brackets in the previous equation correspond respectively to matrices $m_{\Delta-\mathrm{PA}}$ and $m_{\mathrm{R}-\mathrm{PA}}$ obtained with the proposed approach $\left(m_{\Delta-\mathrm{PA}}\right.$ and $m_{\mathrm{R}-\mathrm{PA}}$ are found in Eq. 3$)$. In fact, relation

$$
m_{\mathrm{R}-\mathrm{PA}}=\boldsymbol{R}_{p} \boldsymbol{m}_{\mathrm{R}-\mathrm{LC}}
$$

was used to define $\boldsymbol{R}_{p}\left(\boldsymbol{R}_{p}\right.$ is unique, as $\left.\boldsymbol{R}_{p}=\boldsymbol{m}_{\mathrm{R}-\mathrm{PA}} \boldsymbol{m}_{\mathrm{R}-\mathrm{LC}}^{t}\right)$.

Thereby, we see that $m_{\mathrm{R}-\mathrm{PA}}$ is the composition of two rotations, $\boldsymbol{R}_{p}$ and $m_{\mathrm{R}-\mathrm{LC}}$. We capture the set of all $\boldsymbol{m}_{\mathrm{R}-\mathrm{PA}}$ matrices by allowing $\boldsymbol{R}_{p}$ to vary, where variations in $\boldsymbol{R}_{p}$ stem from variations of $Q_{1}, Q_{2}$, and $\varepsilon$.

Further interpretation. In practice, with a view to minimizing the retardance and using results from section D in the appendix, we do not miss any solution if we restrict the variations of $\boldsymbol{R}_{p}$ to the case $Q_{1}=Q_{2}(=Q)$. Doing this, $\boldsymbol{R}_{p}$ writes
$\boldsymbol{R}_{p}=\boldsymbol{V}^{(p)} \boldsymbol{Q}\left[e^{(p)} \varepsilon\right] \boldsymbol{Q}^{t} \boldsymbol{V}^{(p) t}$. Possible candidates for the diagonal matrix $e^{(p)} \varepsilon$ are (i) the identity, (ii) any matrix among a set of three matrices, comprising one " 1 " and two " -1 "'s on its diagonal. In case (i), $R_{p}$ is the identity and $m_{\mathrm{R}-\mathrm{PA}}=\boldsymbol{m}_{\mathrm{R}-\mathrm{LC}}$. In case (ii), $\boldsymbol{R}_{p}$ is a rotation matrix of angle $\pi$, also called a binary rotation [22].

Remark 1. In the standard case $s_{1}>s_{2}>s_{3}>0, Q_{1}=Q_{2}=$ $\operatorname{diag}([ \pm 1 \pm 1 \pm 1])$, and the $\Delta$ RF set boils down to

$$
\left(\boldsymbol{V}^{(p)} \varepsilon S \boldsymbol{V}^{(p) t}, \boldsymbol{V}^{(p)} \varepsilon \boldsymbol{U}^{(p) t}\right)
$$

There are eight possibilities for $\varepsilon$, among which four match the det $m_{\mathrm{R}}=1$ condition. This is in contrast with the LCGPD, where only one candidate exists, corresponding to $\varepsilon=$ $\operatorname{diag}\left(\left[\begin{array}{lll}1 & 1 & 1\end{array}\right]\right)$ or to $\varepsilon=-\operatorname{diag}\left(\left[\begin{array}{lll}1 & 1 & 1\end{array}\right]\right)$.

Those four options for sign flipping may be linked to sign flipping used in the literature (see e.g., [Eq. 12, 23], or [Eq. 8b, 16]). We defer the corresponding discussion to section 3-C.

Remark 2. In general, eight different cases (one standard case, seven non standard cases) should be distinguished, since there are eight possible configurations for the structure of the three eigenvalues $s_{1}, s_{2}, s_{3}$, each one yielding a specific structure for $Q_{1}$ and $Q_{2}$. The complete study is achieved in section D in the appendix. This is in contrast with the three different non standard cases distinguished in [Appendix B, 8]. Some particular cases in [8] encompass several particular cases of the proposed approach. The standard case in [8] encompasses the standard case and several particular cases of the proposed approach.

For the non standard cases (addressed in [Appendix B, 8]) as for the standard case in [8], we bring forth a larger set of admissible GPD candidates. The final GPD will correspond to the minimizer of the retardance $R$, as in [8]. One consequence is that our methodology may yield a smaller retardance $R$ than the solution from [8].

Remark 3. As already mentioned, we may question whether minimizing the retardance $R$ is the best choice to be made from a physical point of view. For example, in [section 3, 16], the authors make a choice with respect to the orientation of the rotation axis of $m_{R}$, and this may be in contradiction with the minimization of $R$. It should be noted that in this cited reference, the lower retardance (Eq. 20a) as stemming from Eq. 18a is not part of a GPD, since the corresponding $m_{\Delta}$ in Eq. $18 \mathrm{~b}$ is not symmetric. As a consequence, the retardance given in Eq. 20a will not appear in the set of admissible GPD candidates.

\section{B. The $M_{D}$ singular case}

We address here the case $D=1$ [Appendix A, 8]. Matrix $\boldsymbol{M}_{\mathrm{D}}$ being singular, many couples $\left(\boldsymbol{M}_{\Delta}, \boldsymbol{M}_{\mathrm{R}}\right)$ will match $\boldsymbol{M}=$ $\boldsymbol{M}_{\Delta} \boldsymbol{M}_{\mathrm{R}} \boldsymbol{M}_{\mathrm{D}}$. It is recalled (see section 2 and section $\mathrm{A}$ in the appendix) that $M$ writes

$$
\boldsymbol{M}=m_{00}\left[\begin{array}{l}
1 \\
\boldsymbol{P}
\end{array}\right]\left[\begin{array}{ll}
1 & \boldsymbol{D}^{\mathrm{t}}
\end{array}\right]=m_{00}\left[\begin{array}{cc}
1 & \boldsymbol{D}^{\mathbf{t}} \\
\boldsymbol{P} & \boldsymbol{P} \boldsymbol{D}^{\mathrm{t}}
\end{array}\right] .
$$

Thereby, $\boldsymbol{M}$ maps $\left[\begin{array}{l}1 \\ \boldsymbol{D}\end{array}\right]$ to $2 m_{00}\left[\begin{array}{l}1 \\ \boldsymbol{P}\end{array}\right]$.

Besides, considering the SVD of $\boldsymbol{M}_{\mathrm{D}}$ given in section 2, we notice that the range of $\boldsymbol{M}_{\mathrm{D}}$ when $D=1$ is span $\left(\left[\begin{array}{l}1 \\ \boldsymbol{D}\end{array}\right]\right)$. Thereby, with $\boldsymbol{M}=\boldsymbol{M}^{\prime} \boldsymbol{M}_{\mathrm{D}}, \boldsymbol{M}^{\prime}$ is attacked only along span $\left(\left[\begin{array}{l}1 \\ \boldsymbol{D}\end{array}\right]\right)$, and 
the image through $\boldsymbol{M}^{\prime}$ of any vector orthogonal to $\left[\begin{array}{l}1 \\ D\end{array}\right]$ make take any value. This is a degree of freedom in the choice of $\boldsymbol{M}^{\prime}$. Equality $\boldsymbol{M}\left[\begin{array}{l}1 \\ \boldsymbol{D}\end{array}\right]=\boldsymbol{M}^{\prime} \boldsymbol{M}_{\mathrm{D}}\left[\begin{array}{l}1 \\ \boldsymbol{D}\end{array}\right]$ yields $\boldsymbol{M}^{\prime}\left[\begin{array}{l}1 \\ \boldsymbol{D}\end{array}\right]=\left[\begin{array}{l}1 \\ \boldsymbol{P}\end{array}\right]$. This last equation is a necessary and sufficient condition on $\boldsymbol{M}^{\prime}$, which is equivalent to $\boldsymbol{P}=\boldsymbol{P}_{\Delta}+\boldsymbol{m}^{\prime} \boldsymbol{D}$. If we choose $\boldsymbol{P}_{\Delta}=\mathbf{0}$ as in [8], we get $\boldsymbol{P}=\boldsymbol{m}^{\prime} \boldsymbol{D}$ as a necessary and sufficient condition. It is necessary and sufficient for matrix $\boldsymbol{m}^{\prime}$ to map $\boldsymbol{D}$ onto $\boldsymbol{P}$.

The couple

$$
\boldsymbol{M}_{\Delta}=\left[\begin{array}{cc}
1 & \mathbf{0}^{\mathrm{t}} \\
\mathbf{0} & P_{\mathbf{I}}
\end{array}\right], \quad \boldsymbol{M}_{\mathrm{R}}=\left[\begin{array}{cc}
1 & \mathbf{0}^{\mathrm{t}} \\
\mathbf{0} & \mathcal{R}(\boldsymbol{D}, \widehat{\boldsymbol{P}})
\end{array}\right]
$$

is selected in [8]. Matrix $\boldsymbol{m}_{\mathrm{R}}$ rotates $\boldsymbol{D}$ onto $\widehat{\boldsymbol{P}}$, matrix $\boldsymbol{m}_{\Delta}$ maps $\widehat{\boldsymbol{P}}$ onto $\boldsymbol{P}$. As a consequence, matrix $\boldsymbol{m}^{\prime}$ maps $\boldsymbol{D}$ onto $\boldsymbol{P}$.

It should be noticed that if the goal is the minimization of the retardance $R$, the couple

$$
\boldsymbol{M}_{\Delta}=\left[\begin{array}{cc}
1 & \mathbf{0}^{\mathrm{t}} \\
\mathbf{0} & -P_{\mathbf{I}}
\end{array}\right], \quad \boldsymbol{M}_{\mathrm{R}}=\left[\begin{array}{cc}
1 & \mathbf{0}^{\mathrm{t}} \\
\mathbf{0} & \mathcal{R}(\boldsymbol{D},-\widehat{\boldsymbol{P}})
\end{array}\right]
$$

is a better candidate if $\cos (\widehat{\boldsymbol{P}}, \boldsymbol{D})<0$. In this case, $\boldsymbol{D}$ is rotated onto $-\widehat{\boldsymbol{P}}$, and the reflection in $\boldsymbol{m}_{\Delta}$ will ensure that $\boldsymbol{m}^{\prime} \boldsymbol{D}=\boldsymbol{P}$.

Adhering to the principle of [8], the undeterminacy should be solved by minimizing $R$. In fact, $R=0$ and $m_{\mathrm{R}}=\mathbf{I}$ are part of an admissible solution, since there exists a reflection matrix $m_{\Delta}$ which maps $\boldsymbol{D}$ onto $\boldsymbol{P}$.

Let us define vectors $z_{1}, z_{2}, q_{1}, q_{2}, q_{3}$ (the $q_{i}$ 's are unitnormed), and scalars $\alpha_{1}>0$ and $\alpha_{2}>0$ such that (see Fig. 1)

$$
\left\{\begin{array}{l}
\widehat{\boldsymbol{P}}+\widehat{\boldsymbol{D}}=2 z_{1}=\alpha_{1} \boldsymbol{q}_{1}, \\
\widehat{\boldsymbol{P}}-\widehat{\boldsymbol{D}}=2 z_{2}=\alpha_{2} \boldsymbol{q}_{2}
\end{array}\right.
$$

with $\boldsymbol{q}_{3}=q_{1} \times \boldsymbol{q}_{2}$. Thereby $\widehat{\boldsymbol{D}}=z_{1}-z_{2}$ and $\widehat{\boldsymbol{P}}=z_{1}+z_{2}$. Let us define $Q_{\Delta}=\left[\begin{array}{lll}q_{1} & q_{2} & q_{3}\end{array}\right]$ and let us consider $m_{\Delta}=$ $\boldsymbol{Q}_{\Delta} \operatorname{diag}([P-P P]) \boldsymbol{Q}_{\Delta}^{\mathrm{t}}=P \boldsymbol{q}_{1} \boldsymbol{q}_{1}^{\mathrm{t}}-P \boldsymbol{q}_{2} \boldsymbol{q}_{2}^{\mathrm{t}}+P \boldsymbol{q}_{3} \boldsymbol{q}_{3}^{\mathrm{t}}$. We derive: $(\widehat{\boldsymbol{P}}+\widehat{\boldsymbol{D}})^{\mathrm{t}}(\widehat{\boldsymbol{P}}-\widehat{\boldsymbol{D}})=1-1=0$, which yields $z_{1}^{\mathrm{t}} z_{2}=0$ and $\boldsymbol{q}_{1}^{\mathrm{t}} \boldsymbol{q}_{2}=0$.

This gives

$$
\left\{\begin{array}{l}
q_{1} q_{1}^{\mathrm{t}} \widehat{D}=q_{1} q_{1}^{\mathrm{t}}\left(z_{1}-z_{2}\right)=z_{1}, \\
q_{2} q_{2}^{\mathrm{t}} \widehat{D}=q_{2} q_{2}^{\mathrm{t}}\left(z_{1}-z_{2}\right)=-z_{2} .
\end{array}\right.
$$

Finally, $\boldsymbol{m}_{\Delta} \boldsymbol{D}=P z_{1}+P z_{2}=P \widehat{\boldsymbol{P}}=\boldsymbol{P}$ and this particular $m_{\Delta}$ maps $\boldsymbol{D}$ onto $\boldsymbol{P}$. Matrix $m_{\Delta}$ takes the form

$$
m_{\Delta}=P\left(\boldsymbol{q}_{1} \boldsymbol{q}_{1}^{\mathrm{t}}-\boldsymbol{q}_{2} \boldsymbol{q}_{2}^{\mathrm{t}}+\boldsymbol{q}_{3} \boldsymbol{q}_{3}^{\mathrm{t}}\right)=P\left(\mathbf{I}-2 \boldsymbol{q}_{2} \boldsymbol{q}_{2}^{\mathrm{t}}\right),
$$

which is the composition of a uniform scaling and of a Householder reflection of vector $\boldsymbol{q}_{2}$.

\section{Accounting for physical constraints}

The choice of $\varepsilon$ may sometimes be achieved under physical considerations. Work has been devoted to this topic in the case of Mueller matrices with negative determinants (see e.g., [16] and the references therein). For example, for scattering media, the common choice is $\varepsilon=\operatorname{diag}\left(\left[\begin{array}{ll}1 & 1\end{array}-1\right]\right)$ [16]. Such constraints are naturally accounted for in the proposed methodology.
The methodology in [16] can straightforwardly be linked to the present work. With the notations of the present article, Eq. 10 in [16] reads $\boldsymbol{m}^{\prime}=\boldsymbol{V}^{(p)} \boldsymbol{S} \boldsymbol{U}^{(p)^{\prime}}$, Eq. 15a reads $\boldsymbol{m}_{\mathrm{R}}=\boldsymbol{V}^{(p)} \varepsilon \boldsymbol{U}^{(p) t}$, Eq. 15 b reads $m_{\Delta}=\boldsymbol{V}^{(p)} S \varepsilon \boldsymbol{V}^{(p) t}$. It should be noticed that the derivations in [16] are different from ours, and do not address cases different than the case labeled (a) in the present work. In the case of matrix $M$ considered in [16] (see Eq. 16), both solutions coincide: our (unconstrained) approach yields a GPD where $\varepsilon=\operatorname{diag}\left(\left[\begin{array}{ll}1 & 1-1])\end{array}\right)\right.$ and yields $\boldsymbol{M}_{\mathrm{R}}$ and $\boldsymbol{M}_{\Delta}$ found in Eqs. 19a and 19b. The LC-GPD, which selects $\varepsilon=-$ I, yields $R=165$ degrees, instead of $R=79$ degrees with [16] and with the present approach. Nevertheless, imposing $\varepsilon$ on physical grounds does not always give the lowest possible retardance, as will be seen in section 4 .

We propose here a unified framework, where positivedeterminant Mueller matrices are decomposed with the same approach as negative-determinant ones, for diagonal as well as non diagonal depolarizers, as opposed to previous works where those cases where distinguished (see e.g., [16] and the references therein).

\section{Application on synthetic Mueller matrices}

To supplement the preceding derivations, we propose two applications. The first application addresses the standard case $\left(\boldsymbol{M}_{\mathrm{D}}\right.$ invertible, $s_{1}>s_{2}>s_{3}>0$ (case (a)), the second application addresses comparisons with [Appendix B, 8].

First application. We draw random matrices $m_{\Delta}$ and $m_{\mathrm{R}}$ as part of the process of generating a Mueller matrix from elementary components, and devise $\boldsymbol{m}^{\prime}=m_{\Delta} \boldsymbol{m}_{\mathrm{R}}$ (see section $\mathrm{E}$ in the appendix for the algorithm used to generate random Mueller matrices). Minimizing the retardance $R$ amounts to maximizing the trace of $m_{\mathrm{R}}$, since $\operatorname{Tr}\left(\boldsymbol{m}_{\mathrm{R}}\right)=1+2 \cos R$ (see [Eq. 15, 8]). The only degree of freedom for the maximization of the trace is the choice of $\varepsilon$. We get three different such $\varepsilon$ : $\varepsilon_{\mathrm{GT}}$, corresponding to the ground truth, stemming from the underlying generated data; $\varepsilon_{\mathrm{LC}}$, corresponding to the Lu and Chipman solution; and $\varepsilon_{\mathrm{PA}}$, corresponding to the proposed approach.

Examining the simulation outcomes, we see that all possibilities of binary vectors appear for $\varepsilon_{\mathrm{GT}}$ and for $\varepsilon_{\mathrm{PA}}$. This shows that all binary vectors are physically admissible, since all possible binary vectors appear in $\varepsilon_{\mathrm{GT}}$.

In section $\mathrm{F}$ in the appendix, we show that all possibilities of binary vectors may effectively appear for $\varepsilon_{\mathrm{GT}}$. Therefore, for a given matrix $\boldsymbol{m}^{\prime}$, there are four possible binary vectors, among which anyone may be the ground truth. From a purely numerical perspective, nothing allows to distinguish the ground truth for the other binary vectors. Selecting the ground truth among the possibilities should therefore be achieved using physical arguments.

We see that the ground truth $\varepsilon_{\mathrm{GT}}$ does not always minimize the retardance $R$, and that the retardance from [8] is never smaller than the retardance from the proposed approach (see Fig. 2). Vector $\varepsilon_{\mathrm{GT}}$ reaches the minimal retardance in about $55 \%$ of all cases, whereas vector $\varepsilon_{\mathrm{LC}}$ reaches the minimal retardance in about $27 \%$ of all cases. Of course, those rates depend on the probability laws used to generate the data.

Second application. The goal here is to assess [Appendix B, 8]. We draw random matrices $m_{\Delta}$ and $m_{\mathrm{R}}$ as above, but enforce the conditions corresponding to cases (b) to (h). Cases (b) to (d) correspond to the standard case in [8]. Cases (e) and (f) correspond to the case $s_{1} \neq 0, s_{2} \neq 0, s_{3}=0$ in [8]. Case (g) 


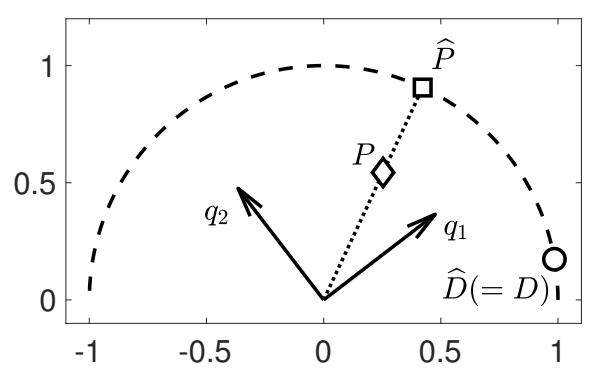

Fig. 1. Mapping of vector $\boldsymbol{D}$ onto vector $\widehat{\boldsymbol{P}}$ by a Householder reflection of vector $\boldsymbol{q}_{2}$. Represented here is the plane defined by vectors $\boldsymbol{D}$ and $\widehat{\boldsymbol{P}}$. The ambiant space is $\mathbb{R}^{3}$. Vectors $\boldsymbol{q}_{1}$ and $\boldsymbol{q}_{2}$ are not represented the right scale (they are unit norm vectors, as $\boldsymbol{D}$ and $\widehat{\boldsymbol{P}}$ are). Vector $\boldsymbol{q}_{3}$ comes out of the figure, as $\boldsymbol{q}_{3}=\boldsymbol{q}_{1} \times \boldsymbol{q}_{2}$, and is not represented.

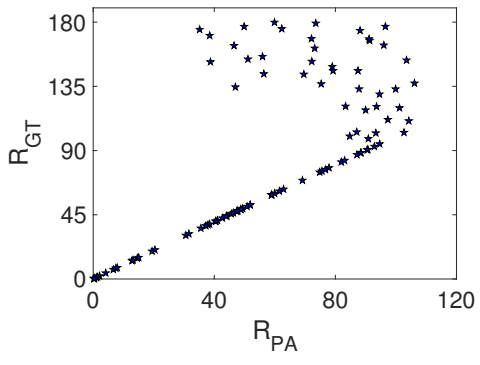

(A)

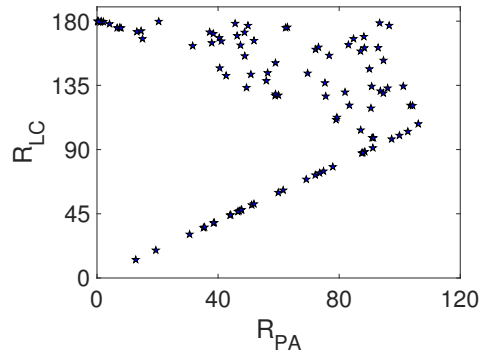

(B)

Fig. 2. Comparison of retardances (in degrees), for 100 randomly simulated matrices $\boldsymbol{m}^{\prime}$, in case (a): $s_{1}>s_{2}>s_{3}>0$. One realization of matrix $m^{\prime}$ appears twice, once in each subplot. (A): retardance $R_{\mathrm{GT}}$ of the ground truth versus retardance $R_{\mathrm{PA}}$ of the proposed approach, (B): retardance $R_{\mathrm{LC}}$ of the Lu and Chipman solution versus retardance $R_{\mathrm{PA}}$ of the proposed approach.

corresponds to the case $s_{1} \neq 0, s_{2}=s_{3}=0$ in [8]. It appears (see Fig. 3) that the solution from [8] does not always yield the minimal retardance, for any of the last two cases addressed in [Appendix B, 8]. The first case of [Appendix B, 8], where $\boldsymbol{m}^{\prime}=\mathbf{0}$, is not assessed here, its solution being trivial.

Comments on Fig. 2 and on Fig. 3 . Let $m_{\mathrm{R}-\mathrm{LC}}$ be the Lu and Chipman solution, and $m_{\mathrm{R}-\mathrm{PA}}$ be the proposed approach solution. Let $R_{\mathrm{LC}}$ and $R_{\mathrm{PA}}$ be the corresponding rotation angles.

- as expected, we always have $R_{\mathrm{LC}} \geqslant R_{\mathrm{PA}}$, and for each case there are samples such that $R_{\mathrm{LC}}>R_{\mathrm{PA}}$;

- we always have $R_{\mathrm{PA}} \leqslant 120$ degrees.

This may be justified as follows. Rotation $m_{\mathrm{R}-\mathrm{PA}}$ writes $m_{\mathrm{R}-\mathrm{PA}}=\boldsymbol{V}^{(p)} \widehat{Q}_{1} \widehat{\varepsilon} \widehat{Q}_{2}^{\mathrm{t}} \boldsymbol{U}^{(p) t}$, which we rewrite $\widehat{V} \widehat{\varepsilon} \widehat{\boldsymbol{U}^{\mathrm{t}}}$, with $\widehat{V}=\boldsymbol{V}^{(p)} \widehat{\boldsymbol{Q}}_{1}$ and $\widehat{\boldsymbol{U}}=\boldsymbol{U}^{(p)} \widehat{\boldsymbol{Q}}_{2}$. The hat notation recalls that $\left(\widehat{Q}_{1}, \widehat{Q}_{2}, \widehat{\varepsilon}\right)$ are resulting from an optimization procedure.

Defining indices $a, b, c \in\{1,2,3\}$ such that

$$
\left|<\boldsymbol{v}_{a}, \boldsymbol{u}_{a}>\right| \geqslant\left|<\boldsymbol{v}_{b}, \boldsymbol{u}_{b}>\right| \geqslant\left|<\boldsymbol{v}_{c}, \boldsymbol{u}_{c}\right\rangle \mid,
$$

we have, as a consequence of $\widehat{\varepsilon}$ being the maximizer,

$$
\begin{aligned}
\operatorname{Tr} \boldsymbol{m}_{\mathrm{R}-\mathrm{PA}} & =\widehat{\boldsymbol{\varepsilon}}_{a}<\boldsymbol{v}_{a}, \boldsymbol{u}_{a}>+\widehat{\boldsymbol{\varepsilon}}_{b}<\boldsymbol{v}_{b}, \boldsymbol{u}_{b}>+\widehat{\boldsymbol{\varepsilon}}_{c}<\boldsymbol{v}_{c}, \boldsymbol{u}_{c}> \\
& =\left|<\boldsymbol{v}_{a}, \boldsymbol{u}_{a}>\right|+\left|<\boldsymbol{v}_{b}, \boldsymbol{u}_{b}>\right|+\widehat{\boldsymbol{\varepsilon}}_{c}<\boldsymbol{v}_{c}, \boldsymbol{u}_{c}>
\end{aligned}
$$

Since

$\left|<\boldsymbol{v}_{b}, \boldsymbol{u}_{b}>\right|+\boldsymbol{\varepsilon}_{c}<\boldsymbol{v}_{c}, \boldsymbol{u}_{c}>\geqslant\left|<\boldsymbol{v}_{b}, \boldsymbol{u}_{b}>\right|-\left|<\boldsymbol{v}_{c}, \boldsymbol{u}_{c}>\right| \geqslant 0$, we have Tr $m_{\mathrm{R}-\mathrm{PA}} \geqslant 0$, and since $\operatorname{Tr} m_{\mathrm{R}-\mathrm{PA}}=1+2 \cos R_{\mathrm{PA}}$, we get $R_{\mathrm{PA}} \leqslant 120$ degrees;

- for each case, there is an exclusion zone defined by the three points $\{(0,0),(90,90),(0,180)\}$ : no sample lies inside this triangle. In others words, if $\boldsymbol{m}_{\mathrm{R}-\mathrm{LC}} \neq \boldsymbol{m}_{\mathrm{R}-\mathrm{PA}}$, then $R_{\mathrm{LC}} \geqslant$ $180-R_{\mathrm{PA}}$.

This may be justified as follows. The analysis boils down to the case $Q_{1}=Q_{2}(=Q)$ : for cases (a)-(d), $Q_{1}=Q_{2}$ stems from the SVD; for cases (e)-(h), $Q_{1}=Q_{2}$ is a consequence of the optimization procedure (see section $\mathrm{D}$ in the appendix). We write $\boldsymbol{m}_{\mathrm{R}-\mathrm{PA}}=\boldsymbol{V}^{(p)} \widehat{\boldsymbol{Q}} \widehat{\mathcal{\varepsilon}} \widehat{\boldsymbol{Q}}^{\mathrm{t}} \boldsymbol{U}^{(p) t}$.

Cases (a)-(d) correspond to the stardard case in [8], where $\boldsymbol{m}_{\mathrm{R}-\mathrm{LC}}=e^{(p)} \boldsymbol{V}^{(p)} \boldsymbol{U}^{(p) t}=e^{(p)} \boldsymbol{V}^{(p)} \widehat{\boldsymbol{Q}} \widehat{\boldsymbol{Q}}^{\mathrm{t}} \boldsymbol{U}^{(p) t}=$ $\boldsymbol{V}^{(p)} \widehat{\boldsymbol{Q}} \varepsilon_{\mathrm{LC}} \widehat{\boldsymbol{Q}}^{\mathrm{t}} \boldsymbol{U}^{(p) t}$, with $\varepsilon_{\mathrm{LC}}=e^{(p)} \mathbf{I}$.

Cases (e)-(f) correspond to the third case in [Appendix B, 8], where $\boldsymbol{m}_{\mathrm{R}-\mathrm{LC}}=\boldsymbol{V}^{(p)} \varepsilon_{\mathrm{LC}} \boldsymbol{U}^{(p) t}$, with $\varepsilon_{\mathrm{LC}}=\operatorname{diag}\left(\left[\begin{array}{ll}1 & 1 \\ 1 & \pm 1\end{array}\right]\right.$, $\varepsilon_{\mathrm{LC}}$ being fixed by the condition det $m_{\mathrm{R}-\mathrm{LC}}=1$. More precisely, for case (e), $\widehat{Q}=\mathcal{R}\left(\boldsymbol{e}_{z}, \widehat{\alpha}\right)$ and $\widehat{Q} \varepsilon_{\mathrm{LC}} \widehat{\boldsymbol{Q}}^{\mathrm{t}}=\varepsilon_{\mathrm{LC}}$ whereas for case (f), $\widehat{Q}$ is diagonal and matches $\widehat{Q} \varepsilon_{\mathrm{LC}} \widehat{Q}^{\mathrm{t}}=$ $\varepsilon_{\mathrm{LC}}$. Finally, we get $\boldsymbol{m}_{\mathrm{R}-\mathrm{LC}}=\boldsymbol{V}^{(p)} \widehat{\boldsymbol{Q}} \varepsilon_{\mathrm{LC}} \widehat{\boldsymbol{Q}}^{\mathrm{t}} \boldsymbol{U}^{(p) t}$

See the Remark below for case (g).

For cases (a)-(f), we may then write

$$
\left\{\begin{array}{l}
m_{\mathrm{R}-\mathrm{LC}}=\boldsymbol{V}^{(p)} \widehat{\boldsymbol{Q}} \varepsilon_{\mathrm{LC}} \widehat{\boldsymbol{Q}}^{\mathrm{t}} \boldsymbol{U}^{(p) t}, \\
m_{\mathrm{R}-\mathrm{PA}}=\boldsymbol{V}^{(p)} \widehat{\boldsymbol{Q}} \varepsilon_{\mathrm{PA}} \widehat{\boldsymbol{Q}}^{\mathrm{t}} \boldsymbol{U}^{(p) t} .
\end{array}\right.
$$


Let us define $\varepsilon_{m}$ such that $\varepsilon_{\mathrm{LC}}=\varepsilon_{m} \varepsilon_{\mathrm{PA}}$. Thereby, we may write

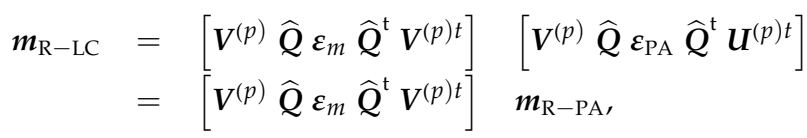

which reads $\boldsymbol{R}_{3}=\boldsymbol{R}_{2} \boldsymbol{R}_{1}$. Let $\left(\boldsymbol{w}_{i}, \theta_{i}\right)$ be the parameters associated to rotation $\boldsymbol{R}_{i}$. If $\boldsymbol{m}_{\mathrm{R}-\mathrm{LC}} \neq \boldsymbol{m}_{\mathrm{R}-\mathrm{PA}}$, then $\varepsilon_{m} \neq \mathbf{I}$, and since det $\boldsymbol{R}_{2}=\operatorname{det} \varepsilon_{m}=1$, $\operatorname{Tr} \varepsilon_{m}=-1$. Thereby, $\theta_{2}=$ $\pi$ and as a consequence (see section $C$ in the appendix):

$$
\cos \frac{\theta_{3}}{2}=\left|\sin \frac{\theta_{1}}{2}\left\langle w_{1}, w_{2}\right\rangle\right| \text {. }
$$

This yields $\cos \frac{\theta_{3}}{2} \leqslant\left|\sin \frac{\theta_{1}}{2}\right|$, which gives

$$
\cos \frac{\theta_{3}}{2} \leqslant \sin \frac{\theta_{1}}{2}\left(=\cos \left(\frac{\pi}{2}-\frac{\theta_{1}}{2}\right)\right) .
$$

It is recalled that $\theta_{1} \in[0, \pi]$ and $\theta_{3} \in[0, \pi]$, since $\theta_{1}$ and $\theta_{3}$ are rotation angles. Thereby, $\theta_{3} \geqslant \pi-\theta_{1}$. QED.

Remark: the analysis above does not apply to case (g), since in this case, and only in this case, $\boldsymbol{V}^{(p)}$ and $\boldsymbol{U}^{(p)}$ in $\boldsymbol{m}_{\mathrm{R}-\mathrm{PA}}$ are user-chosen and therefore may not correspond to the matrices $\boldsymbol{V}^{(p)}$ and $\boldsymbol{U}^{(p)}$ used in $\boldsymbol{m}_{\mathrm{R}-\mathrm{LC}}$. The particular structure of Fig. 3-(g) is justified in section D in the appendix;

- the particular structure of Fig. 3-(d) is justified in section D in the appendix.

\section{THE GENERALIZED POLAR DECOMPOSITION IN PRACTICE - ANALYSIS OF A BIOLOGICAL SAMPLE}

In practice, no singular value will ever vanish, and two singular values will never be strictly equal due to roundoff errors. We let the user define a threshold $\tau$ under which a singular value will be considered vanishing. If the absolute difference between two singular values lies under the same threshold $\tau$, those singular values will be considered equal. Matrix $\boldsymbol{M}_{\mathrm{D}}$ will be considered invertible if and only if $1-D>\tau$.

This procedure is used for three decompositions (LC-GPD, OV-GPD (Ossikovski and Vizet, [16]), and PA-GPD), to analyze a biological sample (see Fig. 4). The sample corresponds to mouse skin measured in vivo at $810 \mathrm{~nm}$. This measurement has been acquired as part of a larger study for the monitoring with multi-spectral polarimetric imaging of grafted tumors [24]. As in [16], the chosen sample contains a zone of pixels with negativedeterminant Mueller matrices. Fig. 4 -(d) shows the $m_{00}$ map of the imaged area, the region of interest being delimited by a black box. The results in terms of retardance maps of the region of interest are given Fig. 4-(a-c). We used $\tau=10^{-8}$.

Negative-determinant pixels are easily spotted in the LCGPD retardance map (see Fig. 4-(a)) as they are characterized by very high values (close to 180 degrees) in contrast with the low retardance values normally observed with mouse skin. As expected, at these locations on the sample the retardance is significantly reduced in OV-GPD and PA-GPD, as compared to LC-GPD. For a more quantitative comparison, a profile of the retardance values along the red line in Fig. 4-(d) is shown for all three decomposition methods in Fig. 4-(e). While in many cases the OV-GPD (i.e., taking $\varepsilon_{\mathrm{OV}}=\operatorname{diag}\left[\begin{array}{ll}1 & 1\end{array}-1\right]$ ) yields results similar to those of the proposed approach, it gives even larger values of retardance than LC-GPD in some cases. Hence, a reduction in retardance is gained from OV-GPD to PA-GPD.

Besides, the $R_{\mathrm{PA}}$ retardance map is smoother than the $R_{\mathrm{LC}}$ map, and also smoother than the $R_{\mathrm{OV}}$ map (see Fig. 4). Smoothness and continuity arguments may be at stake to select a decomposition (see e.g., [16], for the particular case of negativedeterminant Mueller matrices).

As already stated (see section 3-A), the PA-GPD aims at minimizing the retardance. This goal is reached. Other goals may be aimed at, such as considerations on the orientation of the rotation axis, or continuity considerations on given parameters, such as $\boldsymbol{M}_{\Delta}$ [16]. Effectively, abrupt transitions will probably not correspond to any underlying biological reality, and exhibiting non smooth parameter maps is an issue in this context. Generally speaking, smoother maps will probably correspond to more pertinent descriptions of biological tissues. Abrupt changes could therefore be attributed to modeling artifacts or issues, such as minimizing the retardance on a reduced set of candidates. If avoiding discontinuities should undoubtedly be aimed at, perfect smoothness (i.e., constant maps) should definitely be avoided, since we aim at being sensitive to the underlying scene. Indeed, one goal is to use the extracted GPD as features for classification algorithms, with a view to early diagnosis on biological tissues (see e.g., [15]). As a consequence, reducing the roughness of given parameter maps beyond some degree should be carried out only in the light of classification performances. Besides, roughness reduction should also be handled along with the estimation of Mueller matrices from raw measurement images, where noise disturbs the estimates: reducing roughness also comes partly from the estimation procedures retained.

\section{CONCLUSION}

In this article, we revisit the classical Lu and Chipman GPD of Mueller matrices [8]. We devise the entire set of GPD candidates attached to a Mueller matrix. We show that the effective set of GPD candidates is richer than the set considered in the original work [8], and recover the decomposition selected in the literature on physical grounds for negative-determinant Mueller matrices [16].

In [8], the choice among different GPD candidates is achieved by minimizing the retardance. We applied the same principle, and as a result, achieved a better minimization of the retardance. This is illustrated on synthetic Mueller matrices, as well as on a biological sample. On the biological sample, the proposed retardance map proved to be smoother, which is a desirable property in the context of analyzing biological tissues.

Other criteria than the minimization of the retardance on the set of GPD candidates may also be considered. This is left for future work.

\section{APPENDIX}

\section{A. Derivation of $M$ in the $D=1$ case}

If $\boldsymbol{M}_{\mathrm{D}}$ is singular $(D=1)$, then $\boldsymbol{M}=m_{00}\left[\begin{array}{cc}1 & \boldsymbol{D}^{\mathrm{t}} \\ \boldsymbol{P} & \boldsymbol{P} \boldsymbol{D}^{\mathrm{t}}\end{array}\right]$. This expression may be found in [Eq. A3, 8] and in [Eq. 234, 9]. We propose to derive it here, with an algebraic approach, because of the insight given. This derivation is not given in the cited references.

We use the SVD $\left(\boldsymbol{V}_{\mathrm{D}}, \operatorname{diag}\left(S_{\mathrm{D}}\right), \boldsymbol{V}_{\mathrm{D}}\right)$ of $\boldsymbol{M}_{\mathrm{D}}$ (see section 2$)$. With $\boldsymbol{M}=\boldsymbol{M}_{\Delta} \boldsymbol{M}_{\mathrm{R}} \boldsymbol{M}_{\mathrm{D}}$, and $\boldsymbol{M}=m_{00}\left[\begin{array}{cc}1 & \boldsymbol{D}^{\mathrm{t}} \\ \boldsymbol{P} & \boldsymbol{m}\end{array}\right], \boldsymbol{M}_{\mathrm{D}}\left[\begin{array}{c}1 \\ -\widehat{\boldsymbol{D}}\end{array}\right]=\mathbf{0}$ 


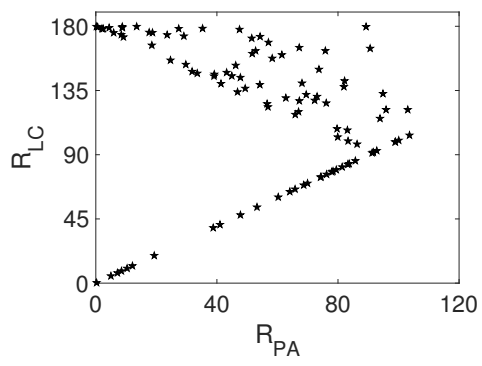

(b): $s_{1}=s_{2}>s_{3}>0$

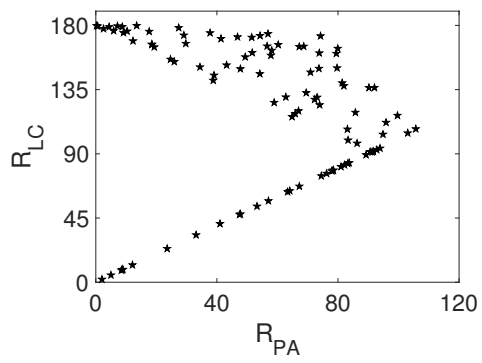

(e): $s_{1}=s_{2}>s_{3}=0$

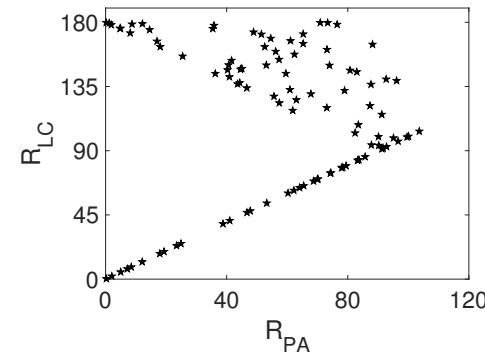

(c): $s_{1}>s_{2}=s_{3}>0$

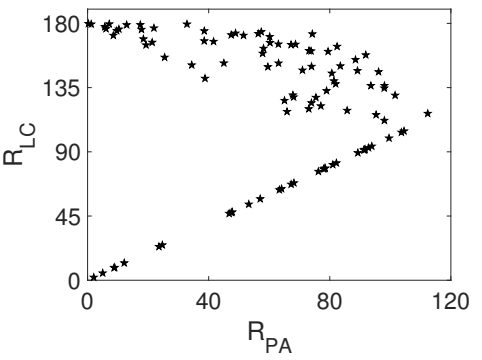

(f): $s_{1}>s_{2}>s_{3}=0$

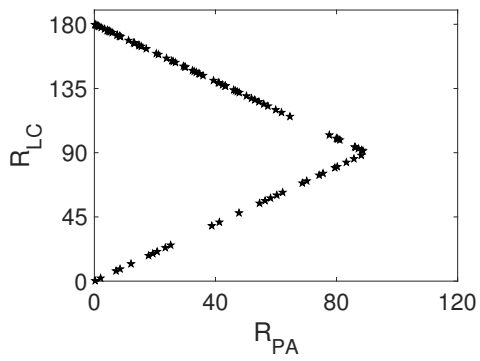

(d): $s_{1}=s_{2}=s_{3}>0$

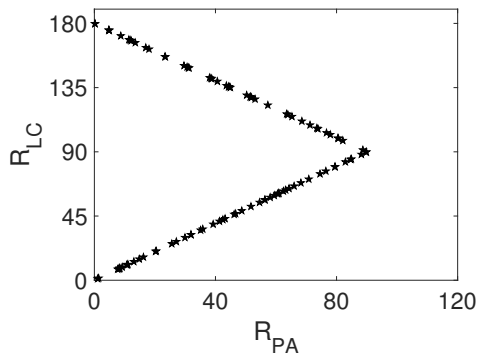

$(\mathrm{g}): s_{1}>s_{2}=s_{3}=0$

Fig. 3. Comparison of retardances (in degrees), for randomly simulated matrices $m^{\prime}$. Cases (b), (c), (d), (e), (f), and (g), 100 samples for each case. The retardance $R_{\mathrm{LC}}$ from the Lu and Chipman approach is plotted against the retardance $R_{\mathrm{PA}}$ from the proposed approach. It may be noticed that, as expected, $R_{\mathrm{PA}}$ is lower than $R_{\mathrm{LC}}$ across all cases, for all samples. The particular structure of (d) and (g) is justified in the appendix (section D). Generally speaking, the structure of all subfigures is justified in the Comments on figures part, in section 3-D.

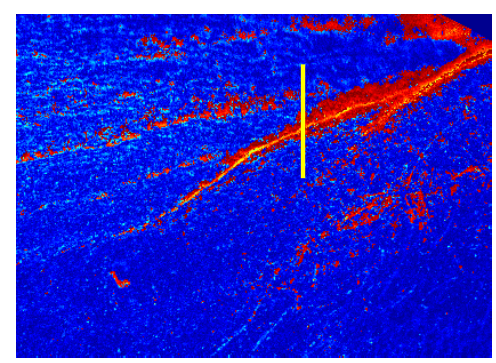

(a) $R_{\mathrm{LC}}$

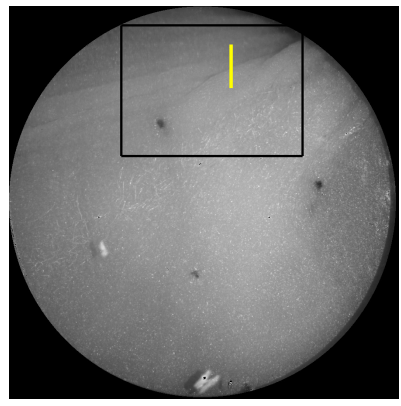

(d)

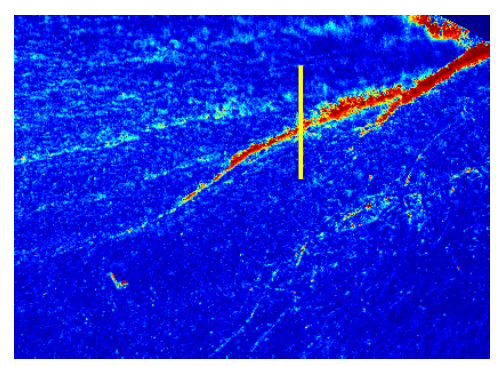

(b) $R_{\mathrm{OV}}$

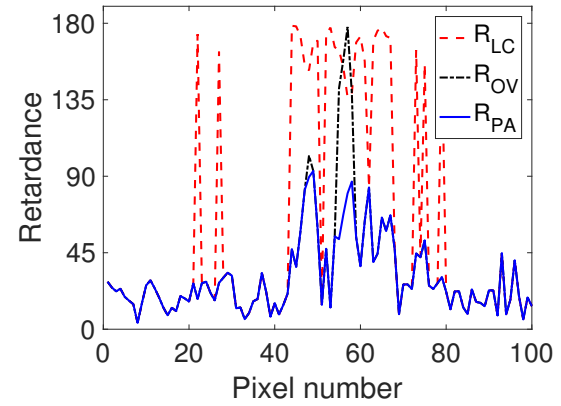

(e)
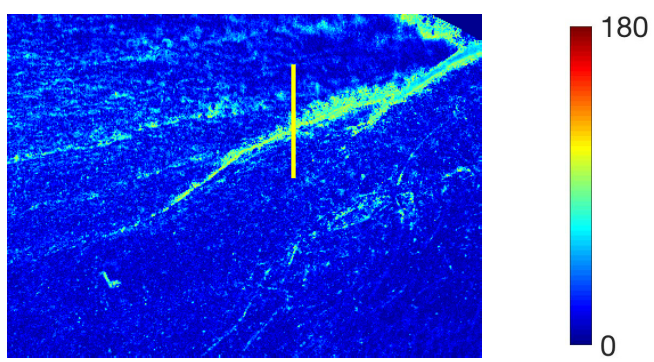

(c) $R_{\mathrm{PA}}$

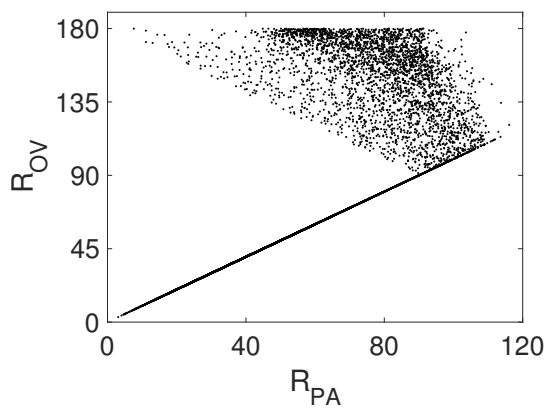

(f)

Fig. 4. Analysis of mouse skin. (d): $m_{00}$ map of the imaged area. The region of interest is delimited by the black box; (a), (b), (c): retardance maps in the region of interest, using the three different approaches $\left(R_{\mathrm{LC}}\right.$ : retardance stemming from the Lu and Chipman approach, $R_{\mathrm{OV}}$ : retardance stemming from the Ossikovski and Vizet approach, $R_{\mathrm{PA}}$ : retardance stemming from the proposed approach); (e): the three retardances plotted against the pixel number, from top (pixel number 1) to bottom (pixel number 100) along the yellow segment appearing in subplots (a)-(d); (f): scatterplot of $R_{\mathrm{OV}}$ against $R_{\mathrm{PA}}$, for negative-determinant pixels in the region of interest. 
yields $\boldsymbol{M}\left[\begin{array}{c}1 \\ -\widehat{\boldsymbol{D}}\end{array}\right]=\mathbf{0}$, which in turn yields $\boldsymbol{P}=\boldsymbol{m} \widehat{\boldsymbol{D}}$ (see also Eq. 1, with $D=1$ ). Finally, we obtain

$$
\boldsymbol{M}\left[\begin{array}{c}
1 \\
\widehat{\boldsymbol{D}}
\end{array}\right]=m_{00}\left[\begin{array}{l}
1+\boldsymbol{D}^{\mathrm{t}} \widehat{\boldsymbol{D}} \\
\boldsymbol{P}+\boldsymbol{m} \widehat{\boldsymbol{D}}
\end{array}\right]=2 m_{00}\left[\begin{array}{l}
1 \\
\boldsymbol{P}
\end{array}\right] .
$$

Since $M_{\mathrm{D}}$ is a rank-1 matrix, $M$ is also a rank-1 matrix, and using Eq. 4 , we conclude that $M$ writes

$$
\boldsymbol{M}=m_{00}\left[\begin{array}{l}
1 \\
\boldsymbol{P}
\end{array}\right]\left[\begin{array}{cc}
1 & \widehat{\boldsymbol{D}}^{\mathrm{t}}
\end{array}\right]=m_{00}\left[\begin{array}{cc}
1 & \boldsymbol{D}^{\mathrm{t}} \\
\boldsymbol{P} & \boldsymbol{P} \boldsymbol{D}^{\mathrm{t}}
\end{array}\right] .
$$

More generally, if $D=1$ and/or $P=1, M$ writes $m_{00}\left[\begin{array}{cc}1 & \boldsymbol{D}^{\mathrm{t}} \\ \boldsymbol{P} & \boldsymbol{P} \boldsymbol{D}^{\mathrm{t}}\end{array}\right]$ (see [25]).

\section{B. The non unicity of the singular value decomposition}

We address here the non unicity of the SVD. We give theorems 3.1.1 and 3.1.1' of [26]. The reader may also refer to [20] and the references therein.

Theorem 3.1.1 Let $A \in M_{m, n}$ be given, and let $q=$ $\min \{m, n\}$. There is a matrix $\Sigma=\left[\sigma_{i j}\right] \in M_{m, n}$ with $\sigma_{i j}=0$ for all $i \neq j$, and $\sigma_{11} \geq \sigma_{22} \geq \ldots \geq \sigma_{q q} \geq 0$, and there are two unitary matrices $V \in M_{m}$ and $\boldsymbol{W} \in M_{n}$ such that $A=V \boldsymbol{\Sigma} \boldsymbol{W}^{\star}$. If $\boldsymbol{A} \in M_{m, n}(\mathbb{R})$, matrices $\boldsymbol{V}$ and $\boldsymbol{W}$ may taken to be real orthogonal matrices.

Theorem 3.1.1' Under the assumptions of Theorem (3.1.1), suppose that the distinct nonzero singular values of $A$ are $s_{1}>$ $\ldots>s_{k}>0$, with respective multiplicities $\mu_{1}, \ldots, \mu_{k} \geq 1$. Let $\mu_{1}+\ldots+\mu_{k}=r$ and let $\boldsymbol{A}=\boldsymbol{V} \boldsymbol{\Sigma} \boldsymbol{W}^{\star}$ be a given singular value decomposition with $\boldsymbol{\Sigma}=\operatorname{diag}\left(s_{1} \mathbf{I}_{\mu_{1}}, \ldots, s_{k} \mathbf{I}_{\mu_{k}}, \mathbf{0}_{q-r}\right) \in M_{m, n}$. Let $\widehat{V} \in M_{m}$ and $\widehat{W} \in M_{n}$ be given unitary matrices. Then $A=\widehat{V} \Sigma \widehat{W}^{\star}$ if and only if there are unitary matrices $U_{i} \in$ $M_{\mu_{i}}, i=1, \ldots, k, \widetilde{\boldsymbol{V}} \in M_{m-r}$, and $\widetilde{\boldsymbol{W}} \in M_{n-r}$ such that

$$
\widehat{\boldsymbol{V}}=\boldsymbol{V}\left[\boldsymbol{U}_{1} \oplus \ldots \oplus \boldsymbol{U}_{k} \oplus \widetilde{\boldsymbol{V}}\right]
$$

and

$$
\widehat{\boldsymbol{W}}=\boldsymbol{W}\left[\boldsymbol{U}_{1} \oplus \ldots \oplus \boldsymbol{U}_{k} \oplus \widetilde{\boldsymbol{W}}\right] .
$$

Notations: $M_{m, n}$ denotes $m$-by- $n$ complex matrices, $M_{n}$ denotes $n$-by- $n$ complex matrices, $M_{m, n}(\mathbb{F})$ denotes $m$-by- $n$ matrices with entries from $\mathbb{F}$. Matrix $\boldsymbol{B} \oplus \boldsymbol{C}$ is the direct sum (i.e., the blockdiagonal matrix) $\left[\begin{array}{ll}B & 0 \\ 0 & C\end{array}\right]$. Matrix $A^{\star}$ is the Hermitian adjoint of $A \in M_{m, n}(\mathbb{C})$. Matrix $\mathbf{I}_{n}$ is the identity matrix in $M_{n}$.

\section{The composition of 3D rotations}

The composition of two 3D rotations is a 3D rotation. We recall here some relations at stake (see e.g., [section 9.4, 22], from which this section is taken).

Let $\boldsymbol{R}_{3}=\boldsymbol{R}_{2} \boldsymbol{R}_{1}$, with the parameters $\boldsymbol{R}_{i}=\mathcal{R}\left(\boldsymbol{u}_{i}, \theta_{i}\right)$ for the rotation of angle $\theta_{i}$ around unit vector $u_{i}$. Let $\lambda_{i}=\cos \frac{\theta_{i}}{2}$, $\boldsymbol{\Lambda}_{i}=\sin \frac{\theta_{i}}{2} \boldsymbol{u}_{i}$.

Parameters $\left(\lambda_{i}, \boldsymbol{\Lambda}_{i}\right)$ are called the Euler-Rodrigues parameters, and correspond to quaternion components. The parameters combine as

$$
\left\{\begin{array}{l}
\lambda_{3}=\lambda_{1} \lambda_{2}-<\Lambda_{1}, \Lambda_{2}> \\
\Lambda_{3}=\lambda_{1} \Lambda_{2}+\lambda_{2} \Lambda_{1}+\Lambda_{2} \times \Lambda_{1} .
\end{array}\right.
$$

Remark: using the previous expressions, $\lambda_{3}$ may take negative values, corresponding to $\theta_{3}$ out of the range $[0, \pi]$ (i.e., $\theta_{3}>\pi$ ). This is useful in some cases. In the present case, if we want to come out with a rotation parameter in $[0, \pi]$, we may proceed as follows if $\lambda_{3}<0$.

We have $\mathcal{R}\left(\boldsymbol{u}_{3}, \theta_{3}\right)=\mathcal{R}\left(-\boldsymbol{u}_{3},-\theta_{3}\right)=\mathcal{R}\left(-\boldsymbol{u}_{3}, 2 \pi-\theta_{3}\right)=$ $\mathcal{R}\left(-\boldsymbol{u}_{3}, \theta_{3}^{\prime}\right)$, with $\theta_{3}^{\prime}=2 \pi-\theta_{3} \in[0, \pi]$.

If $\left(\lambda_{3}^{\prime}, \Lambda_{3}^{\prime}\right)$ are the parameters of $\mathcal{R}\left(-\boldsymbol{u}_{3}, \theta_{3}^{\prime}\right)$, we get:

$$
\left\{\begin{array}{c}
\lambda_{3}^{\prime}=\cos \frac{\theta_{3}^{\prime}}{2}=-\cos \frac{\theta_{3}}{2}=-\lambda_{3}, \\
\Lambda_{3}^{\prime}=-\sin \frac{\theta_{3}^{\prime}}{2} \boldsymbol{u}_{3}=-\sin \frac{\theta_{3}}{2} \boldsymbol{u}_{3}=-\boldsymbol{\Lambda}_{3} .
\end{array}\right.
$$

Finally, in the case $\lambda_{3}<0,\left(-\lambda_{3},-\Lambda_{3}\right)$ are parameters of a rotation with angle in the range $[0, \pi]$.

In any case, whatever the sign of $\lambda_{3}$, we will consider a rotation for which the cosine of the half-angle will read $\left|\lambda_{1} \lambda_{2}-<\Lambda_{1}, \Lambda_{2}\right\rangle \mid$. This latter relation will be used in the present article, with a view of minimizing a rotation angle, or of maximizing the cosine of its half.

\section{Exhaustive analysis of the $\Delta \mathbf{R F}$, when $D<1$}

We address the case where $D<1: \boldsymbol{m}^{\prime}$ is uniquely defined, and we aim at computing its $\triangle R F$. Eight cases should be considered, each one corresponding to a particular structuration of the eigenvalues, and yielding a particular structure for $Q_{1}$ and $Q_{2}$, where $Q_{1}$ and $Q_{2}$ are such that $m^{\prime}=V^{(p)} Q_{1} \varepsilon S Q_{2}^{t} U^{(p) t}$ :

- case (a): $s_{1}>s_{2}>s_{3}>0\left(\Rightarrow Q_{1}=Q_{2}\right)$;

- case (b): $s_{1}=s_{2}>s_{3}>0\left(\Rightarrow Q_{1}=Q_{2}\right)$;

- case (c): $s_{1}>s_{2}=s_{3}>0\left(\Rightarrow Q_{1}=Q_{2}\right)$;

- case (d): $s_{1}=s_{2}=s_{3}>0\left(\Rightarrow Q_{1}=Q_{2}\right)$;

- case (e): $s_{1}=s_{2}>s_{3}=0$;

- case (f): $s_{1}>s_{2}>s_{3}=0$;

- case (g): $s_{1}>s_{2}=s_{3}=0$;

- case (h): $s_{1}=s_{2}=s_{3}=0$.

For each of these cases, the goal is to identify the triple $\left(\widehat{Q}_{1}, \widehat{Q}_{2}, \widehat{\varepsilon}\right)$, which minimizes the retardance $R$ among all admissible candidates $\left(Q_{1}, Q_{2}, \varepsilon\right)$. Since $\operatorname{Tr}\left(\boldsymbol{m}_{\mathrm{R}}\right)=1+2 \cos R$ (see [Eq. 15, 8]), minimizing the retardance amounts to maximizing the trace of $m_{R}$. To save space, we will not go into details of the resolution of each of those subproblems. Only the main lines will be given.

Once the solution $m_{\mathrm{R}}=\boldsymbol{V}^{(p)} \widehat{\boldsymbol{Q}}_{1} \widehat{\varepsilon} \widehat{\boldsymbol{Q}}_{2}^{\mathrm{t}} \boldsymbol{U}^{(p) t}$ is determined, we derive $m_{\Delta}$ from $m_{\Delta}=m^{\prime} m_{\mathrm{R}}^{\mathrm{t}}$.

In the sequel, all $\varepsilon^{\prime} \mathrm{s}$, whatever their subscript, are taken in $\{-1,1\}$. Vectors $\boldsymbol{e}_{x}, \boldsymbol{e}_{y}$, and $\boldsymbol{e}_{z}$ denote the unit vectors of the canonical basis of $\mathbb{R}^{3}$. As in [8], let $\boldsymbol{u}_{1}, \boldsymbol{u}_{2}, \boldsymbol{u}_{3}$ be the column vectors of $\boldsymbol{U}^{(p)}$, and let $\boldsymbol{v}_{1}, \boldsymbol{v}_{2}, \boldsymbol{v}_{3}$ be the column vectors of $\boldsymbol{V}^{(p)}$.

We use a routine which yields

$$
\widehat{\alpha}=\arg \min _{\alpha} a \cos (2 \alpha)+b \sin (2 \alpha)
$$

in closed form, where $a, b \in \mathbb{R}$ are given. Its usefulness appears in the sequel. 
Algorithmically, we successively consider the four possibilities in $\left(\varepsilon_{1}, \varepsilon_{2}\right) \in\{-1,1\}^{2}$. The third component $\varepsilon_{3}$ is then fixed by the det $m_{\mathrm{R}}=1$ constraint, as: $\varepsilon_{3}=$ $\varepsilon_{1} \varepsilon_{2} \operatorname{det}\left(\boldsymbol{V}^{(p)} \boldsymbol{Q}_{1} \boldsymbol{Q}_{2} \boldsymbol{U}^{(p) t}\right)$.

- case (a): $Q_{1}=Q_{2}$, and both matrices are diagonal (diag $([ \pm 1 \pm 1 \pm 1]))$. They cancel out. The $\Delta \mathrm{RF}$ writes

$$
\left(\boldsymbol{V}^{(p)} \varepsilon S \boldsymbol{V}^{(p) t}, \boldsymbol{V}^{(p)} \varepsilon \boldsymbol{U}^{(p) t}\right) .
$$

Amoung all four candidates for $\varepsilon$, we select the one maximizing the trace of $m_{\mathrm{R}}$.

In practice, with a view to reducing the computational cost, we need not examine all four possibilities. Defining indices $a, b, c \in\{1,2,3\}$ such that

$$
\left.\left.\left|<\boldsymbol{v}_{a}, \boldsymbol{u}_{a}\right\rangle|\geqslant|<\boldsymbol{v}_{b}, \boldsymbol{u}_{b}\right\rangle|\geqslant|<\boldsymbol{v}_{c}, \boldsymbol{u}_{c}\right\rangle \mid,
$$

the trace of $\boldsymbol{V}^{(p)} \varepsilon \boldsymbol{U}^{(p) t}$, which writes

$$
\boldsymbol{\varepsilon}_{a}<\boldsymbol{v}_{a}, \boldsymbol{u}_{a}>+\varepsilon_{b}<\boldsymbol{v}_{b}, \boldsymbol{u}_{b}>+\varepsilon_{c}<\boldsymbol{v}_{c}, \boldsymbol{u}_{c}>,
$$

is maximized for

$$
\left\{\begin{array}{ccl}
\varepsilon_{a}= & \operatorname{sign}\left(<\boldsymbol{v}_{a}, \boldsymbol{u}_{a}>\right), \\
\varepsilon_{b} \quad= & \operatorname{sign}\left(<\boldsymbol{v}_{b}, \boldsymbol{u}_{b}>\right), \\
\boldsymbol{\varepsilon}_{c} & \text { such that } & \operatorname{det}\left(\boldsymbol{V}^{(p)} \boldsymbol{\varepsilon} \boldsymbol{U}^{(p) t}\right)=1 .
\end{array}\right.
$$

In other words, the optimal trace writes

$$
\left|<\boldsymbol{v}_{a}, \boldsymbol{u}_{a}>\right|+\left|<\boldsymbol{v}_{b}, \boldsymbol{u}_{b}>\right|+\varepsilon_{c}<\boldsymbol{v}_{c}, \boldsymbol{u}_{c}>;
$$

- $\operatorname{case}(\mathrm{b})$ : $Q_{1}=Q_{2}=Q=\left[\begin{array}{ccc}\cos \alpha & -\varepsilon_{\alpha} \sin \alpha & 0 \\ \sin \alpha & \varepsilon_{\alpha} \cos \alpha & 0 \\ 0 & 0 & \varepsilon_{0}\end{array}\right]$. Thereby, $Q$ writes $\left[\begin{array}{ccc}\cos \alpha & -\sin \alpha & 0 \\ \sin \alpha & \cos \alpha & 0 \\ 0 & 0 & 1\end{array}\right] \operatorname{diag}\left(\left[\begin{array}{lll}1 & \varepsilon_{\alpha} & \varepsilon_{0}\end{array}\right]\right)$, and $Q=\mathcal{R}\left(\boldsymbol{e}_{z}, \alpha\right) \operatorname{diag}\left(\left[\begin{array}{lll}1 & \varepsilon_{\alpha} & \varepsilon_{0}\end{array}\right]\right)$.

Moreover: $Q \in \mathcal{E} Q^{\mathrm{t}}=\mathcal{R}\left(\boldsymbol{e}_{z}, \alpha\right) \varepsilon \mathcal{R}\left(\boldsymbol{e}_{z},-\alpha\right)$ (the $\operatorname{diag}\left(\left[\begin{array}{lll}1 & \varepsilon_{\alpha} & \varepsilon_{0}\end{array}\right]\right)$ part appears twice and cancels out).

- for $\varepsilon_{1}=1, \varepsilon_{2}=1: Q \varepsilon Q^{\mathrm{t}}=\operatorname{diag}\left(\left[\begin{array}{lll}1 & 1 & \varepsilon_{3}\end{array}\right]\right)$ and

$\left.\operatorname{Tr} \boldsymbol{m}_{\mathrm{R}}=\left\langle\boldsymbol{u}_{1}, \boldsymbol{v}_{1}\right\rangle+\left\langle\boldsymbol{u}_{2}, \boldsymbol{v}_{2}\right\rangle+\varepsilon_{3}<\boldsymbol{u}_{3}, \boldsymbol{v}_{3}\right\rangle$;

- for $\varepsilon_{1}=-1, \varepsilon_{2}=-1$ :

$\operatorname{Tr} \boldsymbol{m}_{\mathrm{R}}=-\left\langle\boldsymbol{u}_{1}, \boldsymbol{v}_{1}\right\rangle-\left\langle\boldsymbol{u}_{2}, \boldsymbol{v}_{2}\right\rangle+\varepsilon_{3}\left\langle\boldsymbol{u}_{3}, \boldsymbol{v}_{3}\right\rangle$;

- for $\varepsilon_{1}=1, \varepsilon_{2}=-1$ :

$$
\begin{aligned}
& \operatorname{Tr} \boldsymbol{m}_{\mathrm{R}}=\cos (2 \alpha)\left(<\boldsymbol{u}_{1}, \boldsymbol{v}_{1}>-<\boldsymbol{u}_{2}, \boldsymbol{v}_{2}>\right) \\
& +\sin (2 \alpha)\left(<\boldsymbol{u}_{2}, \boldsymbol{v}_{1}>+<\boldsymbol{u}_{1}, \boldsymbol{v}_{2}>\right) \\
& +\varepsilon_{3}<\boldsymbol{u}_{3}, \boldsymbol{v}_{3}>.
\end{aligned}
$$

Using the aforementioned routine, $\widehat{\alpha}$ is computed and plugged into the expression of the trace;

$$
\begin{aligned}
& - \text { for } \varepsilon_{1}=-1, \varepsilon_{2}=1: \\
& \operatorname{Tr} \boldsymbol{m}_{\mathrm{R}}=\cos (2 \alpha)\left(-<\boldsymbol{u}_{1}, \boldsymbol{v}_{1}>+<\boldsymbol{u}_{2}, \boldsymbol{v}_{2}>\right) \\
& \quad+\sin (2 \alpha)\left(-<\boldsymbol{u}_{2}, \boldsymbol{v}_{1}>-<\boldsymbol{u}_{1}, \boldsymbol{v}_{2}>\right) \\
& \quad+\varepsilon_{3}<\boldsymbol{u}_{3}, \boldsymbol{v}_{3}>
\end{aligned}
$$

- case (c): this case is very close to case (b), with $Q_{1}=Q_{2}=$ $Q=\left[\begin{array}{ccc}\varepsilon_{0} & 0 & 0 \\ 0 & \cos \alpha & -\varepsilon_{\alpha} \sin \alpha \\ 0 & \sin \alpha & \varepsilon_{\alpha} \cos \alpha\end{array}\right]$

$$
\begin{aligned}
-\operatorname{for} \varepsilon_{2} & =1, \varepsilon_{3}=1: \\
\operatorname{Tr} \boldsymbol{m}_{\mathrm{R}} & =\varepsilon_{1}<\boldsymbol{u}_{1}, \boldsymbol{v}_{1}>+<\boldsymbol{u}_{2}, \boldsymbol{v}_{2}>+<\boldsymbol{u}_{3}, \boldsymbol{v}_{3}>; \\
-\operatorname{for} \varepsilon_{2} & =-1, \varepsilon_{3}=-1: \\
\operatorname{Tr} \boldsymbol{m}_{\mathrm{R}} & =\varepsilon_{1}<\boldsymbol{u}_{1}, \boldsymbol{v}_{1}>-<\boldsymbol{u}_{2}, \boldsymbol{v}_{2}>-<\boldsymbol{u}_{3}, \boldsymbol{v}_{3}>; \\
-\operatorname{for} \varepsilon_{2} & =1, \varepsilon_{3}=-1: \\
\operatorname{Tr} \boldsymbol{m}_{\mathrm{R}} & =\cos (2 \alpha)\left(<\boldsymbol{u}_{2}, \boldsymbol{v}_{2}>-<\boldsymbol{u}_{3}, \boldsymbol{v}_{3}>\right) \\
& +\sin (2 \alpha)\left(<\boldsymbol{u}_{3}, \boldsymbol{v}_{2}>+<\boldsymbol{u}_{2}, \boldsymbol{v}_{3}>\right) \\
& +\varepsilon_{1}<\boldsymbol{u}_{1}, \boldsymbol{v}_{1}>; \\
-\operatorname{for} \varepsilon_{2} & =-1, \varepsilon_{3}=1: \\
\operatorname{Tr} \boldsymbol{m}_{\mathrm{R}} & =\cos (2 \alpha)\left(-<\boldsymbol{u}_{2}, \boldsymbol{v}_{2}>+<\boldsymbol{u}_{3}, \boldsymbol{v}_{3}>\right) \\
& -\sin (2 \alpha)\left(<\boldsymbol{u}_{3}, \boldsymbol{v}_{2}>+<\boldsymbol{u}_{2}, \boldsymbol{v}_{3}>\right) \\
& +\varepsilon_{1}<\boldsymbol{u}_{1}, \boldsymbol{v}_{1}>;
\end{aligned}
$$

- case (d): we have $S=s \mathbf{I}, s>0, \boldsymbol{m}^{\prime}=s \boldsymbol{V}^{(p)} \boldsymbol{U}^{(p) t}, \boldsymbol{Q}_{1}=$ $Q_{2}=Q, m_{\mathrm{R}}=\boldsymbol{V}^{(p)} \boldsymbol{Q} \varepsilon \boldsymbol{Q}^{\mathrm{t}} \boldsymbol{U}^{(p) t}, \boldsymbol{m}_{\Delta}=s \boldsymbol{V}^{(p)} \boldsymbol{Q} \varepsilon \boldsymbol{Q}^{\mathrm{t}} \boldsymbol{V}^{(p) t}$. The trace of $m_{\mathrm{R}}$ must be optimized with respect to $Q$ and $\varepsilon$.

Let $e^{(p)}=\operatorname{det}\left(\boldsymbol{V}^{(p)} \boldsymbol{U}^{(p) t}\right)$. The LC-GPD writes

$$
\left(s e^{(p)} \mathbf{I}, e^{(p)} \boldsymbol{V}^{(p)} \boldsymbol{U}^{(p) t}\right) .
$$

Rotation matrix $m_{\mathrm{R}}$ may be linked to the $\mathrm{Lu}$ and Chipman rotation matrix $\boldsymbol{m}_{\mathrm{R}-\mathrm{LC}}=e^{(p)} \boldsymbol{V}^{(p)} \boldsymbol{U}^{(p) t}$ as

$$
\begin{aligned}
\boldsymbol{m}_{\mathrm{R}} & =\boldsymbol{V}^{(p)} \boldsymbol{Q} \varepsilon \boldsymbol{Q}^{\mathrm{t}} \boldsymbol{U}^{(p) t} \\
& =\left[e^{(p)} \boldsymbol{V}^{(p)} Q \varepsilon \boldsymbol{Q}^{\mathrm{t}} \boldsymbol{V}^{(p) t}\right]\left[e^{(p)} \boldsymbol{V}^{(p)} \boldsymbol{U}^{(p) t}\right] \\
& =\left[e^{(p)} \boldsymbol{V}^{(p)} \boldsymbol{Q} \varepsilon \boldsymbol{Q}^{\mathrm{t}} \boldsymbol{V}^{(p) t}\right] \boldsymbol{m}_{\mathrm{R}-\mathrm{LC} .}
\end{aligned}
$$

Matrix $e^{(p)} \boldsymbol{V}^{(p)} Q \varepsilon \boldsymbol{Q}^{\mathrm{t}} \boldsymbol{V}^{(p) t}$ corresponds to a rotation, and the previous equation reads $\boldsymbol{R}_{3}=\boldsymbol{R}_{2} \boldsymbol{R}_{1}$, with parameters $\left(\boldsymbol{w}_{i}, \theta_{i}\right)$ associated to rotation $\boldsymbol{R}_{i}$.

The goal is now to adjust $R_{2}$, i.e., to find matrix $Q$ and vector $\varepsilon$ satisfying $\operatorname{det} \boldsymbol{m}_{\mathrm{R}}=1$, i.e., $\operatorname{det}(\boldsymbol{\varepsilon})=\operatorname{det}\left(\boldsymbol{V}^{(p)} \boldsymbol{U}^{(p) t}\right)=$ $e^{(p)}$, such that $\theta_{3}$ is minimized.

We notice that $e^{(p)} \varepsilon$ is a diagonal matrix, which is either (i) the identity matrix, or (ii) composed of one "1" and two "- 1 "'s.

In case (i), $Q \varepsilon Q^{t}=e^{(p)} \mathbf{I}$, and $\boldsymbol{m}_{\mathrm{R}}=e^{(p)} \boldsymbol{V}^{(p)} \boldsymbol{U}^{(p) t}$. Therefore, the PA-GPD is identical to the LC-GPD.

In case (ii), $\operatorname{Tr} \boldsymbol{R}_{2}=1+2 \cos \theta_{2}=e^{(p)} \operatorname{Tr} \varepsilon=-1$ (this stems from the property $\operatorname{Tr}(\boldsymbol{A B})=\operatorname{Tr}(\boldsymbol{B} \boldsymbol{A}))$. As a consequence, $\theta_{2}=\pi$, which gives (see section $C$ in the appendix):

$$
\cos \frac{\theta_{3}}{2}=\left|\sin \frac{\theta_{1}}{2}\left\langle\boldsymbol{w}_{1}, \boldsymbol{w}_{2}\right\rangle\right|=\sin \frac{\theta_{1}}{2}\left|\left\langle\boldsymbol{w}_{1}, \boldsymbol{w}_{2}\right\rangle\right| .
$$

We aim at maximizing $\cos \frac{\theta_{3}}{2}$, which amounts to maximizing $\left|\left\langle w_{1}, w_{2}\right\rangle\right|$. This yields $\boldsymbol{w}_{2}= \pm \boldsymbol{w}_{1}$. It may be noticed that both possibilities for $w_{2}$ correspond to the same rotation. In the sequel, we will consider $w_{2}=w_{1}$. Thereby $R_{2}$, and $\boldsymbol{R}_{3}$ as a consequence, are completely defined. In this case, $\cos \frac{\theta_{3}}{2}=\sin \frac{\theta_{1}}{2}$, and $\theta_{3}=\pi-\theta_{1}$.

It remains to be checked whether $w_{2}=w_{1}$, where $w_{1}$ given, is accessible, and to compute $Q \in \mathcal{E} Q^{\mathrm{t}}$. Vector $w_{1}$ being unconstrained, the problem boils down to: given any 
$\boldsymbol{w}_{2}$, find $Q$ and $\varepsilon$ such that the rotation $e^{(p)} \boldsymbol{V}^{(p)} Q \varepsilon Q^{t} \boldsymbol{V}^{(p) t}$ has axis $w_{2}$ (i.e., $w_{2}$ is a vector associated to the eigenvalue 1 of this rotation matrix) and angle $\theta_{2}=\pi$.

Let $z_{a}=V^{(p) t} w_{2}$. The goal is to compute $Q$ and $\varepsilon$ such that $e^{(p)} \boldsymbol{V}^{(p)} Q \varepsilon Q^{\mathrm{t}} \boldsymbol{V}^{(p) t} \boldsymbol{w}_{2}=\boldsymbol{w}_{2}\left(\Leftrightarrow e^{(p)} Q \varepsilon \boldsymbol{Q}^{\mathrm{t}} \boldsymbol{z}_{a}=z_{a}\right)$. Let $\boldsymbol{q}_{a}=z_{a}$, and $\left(\boldsymbol{q}_{b}, \boldsymbol{q}_{c}\right)$ be an orthonormal basis of $\left(\operatorname{span}\left(\boldsymbol{q}_{a}\right)\right)^{\perp}$. We notice that

$$
\left\{\begin{aligned}
Q & =\left[\boldsymbol{q}_{a} \boldsymbol{q}_{b} \boldsymbol{q}_{c}\right] \\
\boldsymbol{\varepsilon} & =e^{(p)} \operatorname{diag}([1-1-1]),
\end{aligned}\right.
$$

meets all conditions. With this choice, we get

$$
Q \varepsilon Q^{t}=e^{(p)}\left(\boldsymbol{q}_{a} \boldsymbol{q}_{a}^{t}-\boldsymbol{q}_{b} \boldsymbol{q}_{b}^{t}-\boldsymbol{q}_{c} \boldsymbol{q}_{c}^{t}\right),
$$

which gives

$$
\begin{aligned}
\boldsymbol{R}_{2} \boldsymbol{w}_{2} & =e^{(p)} \boldsymbol{V}^{(p)} \boldsymbol{Q} \varepsilon \boldsymbol{Q}^{\mathrm{t}} \boldsymbol{V}^{(p) t} \boldsymbol{w}_{2} \\
& =\boldsymbol{V}^{(p)}\left(\boldsymbol{q}_{a} \boldsymbol{q}_{a}^{t}-\boldsymbol{q}_{b} \boldsymbol{q}_{b}^{t}-\boldsymbol{q}_{c} \boldsymbol{q}_{c}^{t}\right) \boldsymbol{q}_{a} \\
& =\boldsymbol{V}^{(p)} \boldsymbol{q}_{a}=\boldsymbol{V}^{(p)} \boldsymbol{z}_{a}=\boldsymbol{w}_{2},
\end{aligned}
$$

and any vector $y$ orthogonal to $w_{2}$ is inverted, i.e., $\pi$ rotated:

$$
e^{(p)} \boldsymbol{V}^{(p)} \boldsymbol{Q} \varepsilon \boldsymbol{Q}^{\mathrm{t}} \boldsymbol{V}^{(p) t} \boldsymbol{y}=-\boldsymbol{y} .
$$

Final algorithm: let $\boldsymbol{m}_{\mathrm{R}-\mathrm{LC}}=e^{(p)} \boldsymbol{V}^{(p)} \boldsymbol{U}^{(p) t}$ be the $\mathrm{Lu}$ and Chipman rotation, parameterized as $\mathcal{R}\left(\boldsymbol{w}_{1}, \theta_{1}\right)$.

If $\theta_{3(i)} \leqslant \theta_{3(i i)}$, i.e., if $\theta_{1} \leqslant \pi-\theta_{1} \Leftrightarrow \theta_{1} \leqslant \pi / 2$ :

$$
m_{\mathrm{R}}=m_{\mathrm{R}-\mathrm{LC}} .
$$

Otherwise, the solution is given by $\boldsymbol{q}_{a}=\boldsymbol{V}^{(p) t} \boldsymbol{w}_{1}$, Eqs. (7), and

$$
m_{\mathrm{R}}=V^{(p)} Q \varepsilon Q^{\mathrm{t}} U^{(p) t}
$$

- case (e): this case is close to case (b), with

$$
\begin{aligned}
& Q_{1}=\left[\begin{array}{ccc}
\cos \alpha & -\sin \alpha & 0 \\
\sin \alpha & \cos \alpha & 0 \\
0 & 0 & 1
\end{array}\right] \operatorname{diag}\left(\left[\begin{array}{lll}
1 & \varepsilon_{\alpha} & \varepsilon_{01}
\end{array}\right]\right), \\
& Q_{2}=\left[\begin{array}{ccc}
\cos \alpha & -\sin \alpha & 0 \\
\sin \alpha & \cos \alpha & 0 \\
0 & 0 & 1
\end{array}\right] \operatorname{diag}\left(\left[\begin{array}{lll}
1 & \varepsilon_{\alpha} & \varepsilon_{02}
\end{array}\right]\right) .
\end{aligned}
$$

It may be shown that det $m_{\mathrm{R}}=1$ yields the condition $\varepsilon_{01} \varepsilon_{02} \varepsilon_{3}=\varepsilon_{1} \varepsilon_{2} \operatorname{det}(\boldsymbol{V}) \operatorname{det}(\boldsymbol{U})$. Once $\varepsilon_{1}$ and $\varepsilon_{2}$ are chosen, the product $\varepsilon_{01} \varepsilon_{02} \varepsilon_{3}$ is fixed. The individual choices for $\varepsilon_{01}, \varepsilon_{02}, \varepsilon_{3}$ do not matter, and $\varepsilon_{\alpha}$ cancels out.

Therefore, we do not miss any solution if we fix $\varepsilon_{01}=\varepsilon_{02}=$ 1 , and let $\varepsilon_{3}$ be imposed by the choices of $\varepsilon_{1}$ and $\varepsilon_{2}$. We are then in the same configuration as the one of case (b): $Q_{1}=Q_{2}=\mathcal{R}\left(e_{z}, \widehat{\alpha}\right)$;

- case (f): this case is close to case (a). We have: $Q_{1}=\operatorname{diag}\left(\left[\varepsilon_{a} \varepsilon_{b} \varepsilon_{c}\right]\right)$ and $Q_{2}=\operatorname{diag}\left(\left[\varepsilon_{a} \varepsilon_{b} \varepsilon_{d}\right]\right)$. The condition det $m_{\mathrm{R}}=1$ yields $\varepsilon_{c} \quad \varepsilon_{d} \varepsilon_{3}=$ $\varepsilon_{1} \varepsilon_{2} \operatorname{det}(\boldsymbol{V}) \operatorname{det}(\boldsymbol{U})$.

Once $\varepsilon_{1}$ and $\varepsilon_{2}$ are chosen, the product $\varepsilon_{c} \varepsilon_{d} \varepsilon_{3}$ is fixed. The individual choices for $\varepsilon_{c}, \varepsilon_{d}, \varepsilon_{3}$ do not matter. We may fix $\varepsilon_{c}=\varepsilon_{d}=1$, and let $\varepsilon_{3}$ be imposed by the choices of $\varepsilon_{1}$ and $\varepsilon_{2}$. We are then in the same configuration as the one of case (a): $Q_{1}=Q_{2}=Q$, which is a diagonal matrix;
- case (g): $\boldsymbol{m}^{\prime}=s_{1} \boldsymbol{v}_{1} \boldsymbol{u}_{1}^{\mathrm{t}}$. We choose $\boldsymbol{u}_{2}=\frac{1}{\left\|\boldsymbol{u}_{1} \times \boldsymbol{v}_{1}\right\|} \boldsymbol{u}_{1} \times \boldsymbol{v}_{1}$, $v_{2}=u_{2}, u_{3}=u_{1} \times u_{2}, v_{3}=v_{1} \times v_{2}$.

In the case where $\left\|u_{1} \times v_{1}\right\|=0$, i.e., $\boldsymbol{u}_{1}= \pm \boldsymbol{v}_{1}$, we choose $u_{2}$ as any vector orthonormal to $v_{1}$ and the sequel still applies.

We now have a particular $\boldsymbol{V}^{(p)}$ and a particular $\boldsymbol{U}^{(p)}$.

We have to optimize the trace of $\boldsymbol{m}_{\mathrm{R}}=\boldsymbol{V}^{(p)} \boldsymbol{Q}_{1} \varepsilon \boldsymbol{Q}_{2}^{\mathrm{t}} \boldsymbol{U}^{(p) t}$ with respect to $Q_{1}, Q_{2}, \varepsilon$. This trace is also $\operatorname{Tr}\left(\left(\boldsymbol{Q}_{1} \varepsilon \boldsymbol{Q}_{2}^{\mathrm{t}}\right) \times\left(\boldsymbol{U}^{(p) t} \boldsymbol{V}^{(p)}\right)\right)$.

Let $\cos \theta=<\boldsymbol{u}_{1}, \boldsymbol{v}_{1}>$. We get:

$$
\boldsymbol{U}^{(p) t} \boldsymbol{V}^{(p)}=\left[\begin{array}{ccc}
\cos \theta & 0 & -\sin \theta \\
0 & 1 & 0 \\
\sin \theta & 0 & \cos \theta
\end{array}\right]
$$

We write $Q_{1}=\left[\begin{array}{ccc}1 & 0 & 0 \\ 0 & \cos \alpha_{1} & -\sin \alpha_{1} \\ 0 & \sin \alpha_{1} & \cos \alpha_{1}\end{array}\right] \operatorname{diag}\left(\left[\begin{array}{lll}\varepsilon_{0} & 1 & \varepsilon_{\alpha_{1}}\end{array}\right]\right)$, and $Q_{2}=\left[\begin{array}{ccc}1 & 0 & 0 \\ 0 & \cos \alpha_{2} & -\sin \alpha_{2} \\ 0 & \sin \alpha_{2} & \cos \alpha_{2}\end{array}\right] \operatorname{diag}\left(\left[\begin{array}{lll}\varepsilon_{0} & 1 & \varepsilon_{\alpha_{2}}\end{array}\right]\right)$.

As seen above, it appears that we can choose $\varepsilon_{\alpha_{1}}=\varepsilon_{\alpha_{2}}=1$ without missing anything. Moreover, variable $\varepsilon_{0}$ cancels out. Since

$$
\operatorname{det}\left(\boldsymbol{U}^{(p) t} \boldsymbol{V}^{(p)}\right)=\operatorname{det}\left(\boldsymbol{U}^{(p)}\right) \operatorname{det}\left(\boldsymbol{V}^{(p)}\right)=1 \times 1=1,
$$

the constraint det $m_{\mathrm{R}}=1$ yields det $\varepsilon=\prod_{i} \varepsilon_{i}=1$.

We distinguish two possibilities:

1. $\varepsilon_{2}=\varepsilon_{3}, \varepsilon_{1}=1$. We get

$$
\operatorname{Tr} \boldsymbol{m}_{\mathrm{R}}=\varepsilon_{3} \cos \left(\alpha_{1}-\alpha_{2}\right)(1+\cos \theta)+\cos \theta .
$$

This trace may be maximized with $\varepsilon_{3}=1, \alpha_{1}=\alpha_{2}=0$. Finally $Q_{1}=Q_{2}=\mathbf{I}, \varepsilon=\mathbf{I}, m_{\mathrm{R}}=\boldsymbol{V}^{(p)} \boldsymbol{U}^{(p) t}$, and $\operatorname{Tr} m_{\mathrm{R}}=1+2 \cos \theta$;

2. $\varepsilon_{2}=-\varepsilon_{3}, \varepsilon_{1}=-1$. We get

$$
\operatorname{Tr} m_{\mathrm{R}}=-\varepsilon_{3} \cos \left(\alpha_{1}+\alpha_{2}\right)(1-\cos \theta)-\cos \theta .
$$

This trace may be maximized with $\varepsilon_{2}=1, \varepsilon_{3}=$ $-1, \alpha_{1}=\alpha_{2}=0$. Finally $Q_{1}=Q_{2}=\mathbf{I}, \varepsilon=$ $\operatorname{diag}([-11-1]), \boldsymbol{m}_{\mathrm{R}}=\boldsymbol{V}^{(p)} \boldsymbol{\varepsilon} \boldsymbol{U}^{(p) t}$, and $\operatorname{Tr} \boldsymbol{m}_{\mathrm{R}}=$ $1-2 \cos \theta$.

The algorithm boils down to:

- if $\cos \theta \geqslant 0$, the solution is $Q_{1}=Q_{2}=\mathbf{I}, \varepsilon=\mathbf{I}$, $\boldsymbol{m}_{\mathrm{R}}=\boldsymbol{V}^{(p)} \boldsymbol{U}^{(p) t}, \operatorname{Tr} \boldsymbol{m}_{\mathrm{R}}=1+2 \cos \theta$;

- if $\cos \theta<0$, the solution is $Q_{1}=Q_{2}=\mathbf{I}, \varepsilon=$ $\operatorname{diag}([-11-1]), m_{\mathrm{R}}=\boldsymbol{V}^{(p)} \varepsilon \boldsymbol{U}^{(p) t}, \operatorname{Tr} \boldsymbol{m}_{\mathrm{R}}=1-$ $2 \cos \theta$.

In other words, if $\cos \theta \geqslant 0, m_{\mathrm{R}}$ is the smallest angle rotation that transfers $u_{1}$ to $v_{1}$, whereas if $\cos \theta<0, m_{R}$ is the smallest angle rotation that transfers $\boldsymbol{u}_{1}$ to $-\boldsymbol{v}_{1}$;

- case (h): $\boldsymbol{m}^{\prime}=\mathbf{0}$, minimizing $R$ yields $R=0, \boldsymbol{m}_{\mathrm{R}}=\mathbf{I}$. 
It should be noticed that cases (e) and (f) are addressed under the same case in [8] (but differently than is done here, and with sometimes a larger $R$ as a result). See [Appendix B, 8], case $s_{1} \neq 0, s_{2} \neq 0, s_{3}=0$.

Case $(\mathrm{g})$ is addressed by [8] (Appendix $\mathrm{B}$, case $s_{1} \neq 0, s_{2}=$ $\left.0, s_{3}=0\right)$. In the original article, $\boldsymbol{u}_{1}$ is always transferred to $\boldsymbol{v}_{1}$. We showed that this is appropriate if $\left.\left\langle\boldsymbol{u}_{1}, \boldsymbol{v}_{1}\right\rangle\right\rangle 0$, but if $\left.<\boldsymbol{u}_{1}, \boldsymbol{v}_{1}\right\rangle<0$, then $\boldsymbol{u}_{1}$ should be transferred to $-\boldsymbol{v}_{1}$ (this is seen in our solution, where $\varepsilon_{1}=-1$ ).

\section{E. Generation of synthetic random Mueller matrices}

In this section, we detail the algorithm used to generate random synthetic Mueller matrices. Those sample matrices are used to enlighten the proposed GPD. Therefore, our Mueller matrix synthesis algorithm mimics the GPD: Mueller matrices are devised starting from elementary components (e.g., $D, m_{\mathrm{D}}, m_{\Delta}$, etc.). The proposed algorithm allows to reach any particular case (e.g., $\boldsymbol{M}_{\mathrm{D}}$ invertible or non invertible, any particular structure (a)-(h), etc.). Besides, any generated sample Mueller matrix is physically admisssible, and any physically admissible Mueller matrix may be reached by our generation algorithm (i.e., there is no blind spot in the Mueller matrix space).

Other random Mueller matrix generation algorithms may be found in the literature (see e.g., [section 7, 27]: brute force generation, [section 5.1.1,28]: generation based on the coherency matrix). But we found none mimicking the GPD, which motivated the development of our own method.

Nine different possibilities stem from $P$ and $D$ : there are three possibilities for $D(D=0, D \in] 0,1[, D=1)$ and three possibilities for $P(P=0, P \in] 0,1[, P=1)$, to be combined (see [p. 38, 9]). Besides, there are eight different configurations for the singular values of $\boldsymbol{m}^{\prime}((\mathrm{a})$ to $(\mathrm{h}))$. Globally, there are $9 \times 8=72$ particular cases, structuring the generation of random Mueller matrices.

For a finer analysis, one could want to distinguish three subcases: $\widehat{\boldsymbol{P}} \neq \pm \widehat{\boldsymbol{D}}, \widehat{\boldsymbol{P}}=\widehat{\boldsymbol{D}}, \widehat{\boldsymbol{P}}=-\widehat{\boldsymbol{D}}$. Such a refinement might be of interest in some configurations (see e.g., Fig. 1).

For any particular case taken from the set of 72 possibilities, a candidate Mueller matrix is drawn using the following procedure:

- draw $P, D, \widehat{\boldsymbol{P}}$, and $\widehat{\boldsymbol{D}}$;

- draw $m_{00}$, which must meet $m_{00}(1+D) \leqslant 1$ and $m_{00}(1+P) \leqslant 1$ (see [eq. 225, 9]);

- devise $\boldsymbol{M}_{\mathrm{R}}$ by drawing $\boldsymbol{m}_{\mathrm{R}}$ (draw the angle and the axis of this $3 \times 3$ rotation matrix);

- compute $\boldsymbol{M}_{\mathrm{D}}$ using $m_{00}$ and $\boldsymbol{D}$;

- draw $M_{\Delta}$ (see below);

- compute the candidate Mueller matrix as the product $\boldsymbol{M}_{\Delta} \boldsymbol{M}_{\mathrm{R}} \boldsymbol{M}_{\mathrm{D}}$;

- accept the candidate if the physical admissibility conditions $\mathcal{C}$ (see below) are met, otherwise restart the procedure.

Matrix $\boldsymbol{M}_{\Delta}$ is drawn as follows (see [section 6, 8]):

- draw $a, b, c$ in $[0,1]$. Redefine $a, b, c$ by sorting the previous values in decreasing order. Modify $a, b, c$ by enforcing the condition on the eigenvalues ((a) to (h)) if necessary. Update $a, b, c$ by randomly flippling their signs and randomly swapping their positions;
- draw a $3 \times 3$ unitary matrix $Q$;

- compute $m_{\Delta}$ as $m_{\Delta}=Q \operatorname{diag}([a b c]) Q^{t}$;

- compute $\boldsymbol{P}_{\Delta}$ using $\boldsymbol{P}=\boldsymbol{P}_{\Delta}+\boldsymbol{m}_{\Delta} \boldsymbol{m}_{\mathrm{R}} \boldsymbol{D}$;

- compute $M_{\Delta}$.

It is reminded that $m^{\prime} m^{\prime t}=m_{\Delta} m_{\Delta}^{t}$ (see section 3-A). Besides, from $m_{\Delta}$ written $m_{\Delta}=Q \operatorname{diag}([a b c]) Q^{t}$, it appears that the singular values of $m_{\Delta}$ are $|a|,|b|,|c|$ (to be taken in decreasing order), and the singular values of $m^{\prime}$ are $s_{1}, s_{2}, s_{3}$. As a consequence, the sets $\{|a|,|b|,|c|\}$ and $\left\{s_{1}, s_{2}, s_{3}\right\}$ are equal, and enforcing a condition on the $s_{i}$ 's amounts to enforcing the same condition on $|a|,|b|,|c|$, which is what is done is the algorithm.

Necessary and sufficient conditions for a $4 \times 4$ real matrix to be a physically admissible Mueller matrix are (see e.g., [9]: see Eqs. 171 and 225 in [9], see [p. 29, (4), 9]):
$(\mathcal{C}) \begin{cases}\left(\mathcal{C}_{1}\right) & m_{00}+\left\|\left[m_{01} m_{02} m_{03}\right]\right\| \leqslant 1 \\ \left(\mathcal{C}_{2}\right) & m_{00}+\left\|\left[m_{10} m_{20} m_{30}\right]\right\| \leqslant 1 \\ \left(\mathcal{C}_{3}\right) & \text { the eigenvalues of } \boldsymbol{H} \text { must be positive, }\end{cases}$

where $\boldsymbol{H}$ is the corresponding coherency matrix. Conditions $\mathcal{C}_{1}$ and $\mathcal{C}_{2}$ respectively read $m_{00}(1+D) \leqslant 1$ and $m_{00}(1+P) \leqslant 1$.

It may be noticed that the condition $D \leqslant 1$, which is used in the algorithm, stems from conditions on the gain (minimum and maximum transmittance, see e.g., [Eq. 27, 8], [Eqs. 3.9 and 3.10, 29]). Besides, condition $P \leqslant 1$ stems from considering the degree of polarization, which must be lower than 1 , in particular in the case of an non polarized incoming light (see e.g., [Eq. 31, 8]).

The proposed algorithm enforces only necessary conditions, and checks whether the candidate results meets conditions $\mathcal{C}$. Any physically admissible Mueller matrix may be reached by our procedure.

\section{F. Identifiability issues}

We consider here being in case (a), $\boldsymbol{M}_{\mathrm{D}}$ being therefore invertible. We show that by flipping two $\varepsilon$ 's, we still get a couple $\left(\boldsymbol{m}_{\Delta}, \boldsymbol{m}_{\mathrm{R}}\right)$ which might have been drawn by the procedure described in section $\mathrm{E}$ just above. The present section is referred to in section 3-D.

Let $\left(m_{\Delta i}, m_{\mathrm{R} i}\right)$ be an initial draw with $\boldsymbol{m}^{\prime}=m_{\Delta i} \boldsymbol{m}_{\mathrm{R} i}$. Matrix $m_{\Delta i}$ is supposed to stem from $\left(Q_{i}, a_{i}, b_{i}, c_{i}\right)$ as $m_{\Delta i}=$ $Q_{i} \operatorname{diag}\left(\left[a_{i} b_{i} c_{i}\right]\right) Q_{i}^{t}$. We write

$$
\begin{aligned}
\operatorname{diag}\left(\left[a_{i} b_{i} c_{i}\right]\right)= & \operatorname{diag}\left(\left[\operatorname{sign}\left(a_{i}\right) \operatorname{sign}\left(b_{i}\right) \operatorname{sign}\left(c_{i}\right)\right]\right) \\
& \times \operatorname{diag}\left(\left[\left|a_{i}\right|\left|b_{i}\right|\left|c_{i}\right|\right]\right) .
\end{aligned}
$$

Defining the permutation matrix $\Pi$ such that the values of $\Pi^{t}$ diag $\left(\left[\left|a_{i}\right|\left|b_{i}\right|\left|c_{i}\right|\right]\right) \Pi$ appear in decreasing order along the diagonal, we may write

$$
\begin{aligned}
m_{\Delta i}= & \boldsymbol{Q}_{i} \boldsymbol{\Pi}\left[\boldsymbol{\Pi}^{t} \operatorname{diag}\left(\left[\operatorname{sign}\left(a_{i}\right) \operatorname{sign}\left(b_{i}\right) \operatorname{sign}\left(c_{i}\right)\right]\right) \boldsymbol{\Pi}\right] \\
& \times\left[\boldsymbol{\Pi}^{t} \operatorname{diag}\left(\left[\left|a_{i}\right|\left|b_{i}\right|\left|c_{i}\right|\right]\right) \boldsymbol{\Pi}\right] \boldsymbol{\Pi}^{t} \boldsymbol{Q}_{i}^{t} .
\end{aligned}
$$

This expression reads as a SVD of $m_{\Delta i}$, in the form $m_{\Delta i}=$ $\boldsymbol{V}^{(p)} \varepsilon \boldsymbol{S} \boldsymbol{V}^{(p) t}$, with

$$
\begin{cases}\boldsymbol{V}^{(p)} & =\boldsymbol{Q}_{i} \boldsymbol{\Pi}, \\ \varepsilon & =\boldsymbol{\Pi}^{t} \operatorname{diag}\left(\left[\operatorname{sign}\left(a_{i}\right) \operatorname{sign}\left(b_{i}\right) \operatorname{sign}\left(c_{i}\right)\right]\right) \boldsymbol{\Pi}, \\ \boldsymbol{S} & =\boldsymbol{\Pi}^{t} \operatorname{diag}\left(\left[\left|a_{i}\right|\left|b_{i}\right|\left|c_{i}\right|\right]\right) \boldsymbol{\Pi} .\end{cases}
$$


The objective is to show that flipping the sign of two $\varepsilon^{\prime}$ s will yield a matrix that may have been drawn from scratch by the general procedure. We flip two $\varepsilon^{\prime}$ 's (as opposed to one or three) as the resulting associated rotation matrix must have a positive determinant. Flipping two $\varepsilon^{\prime}$ s amounts to flipping the sign of two components of $\left[a_{i} b_{i} c_{i}\right]$. Such a flipped vector can be reached by the procedure, and will yield and admissible $m_{\Delta}$ (i.e., symmetrical, with $|a|,|b|,|c| \leqslant 1)$. The rotation matrix $m_{\mathrm{R}}$ would be modified, but would still be an admissible rotation matrix, which as a consequence could be reached by the procedure, since the procedure may reach any rotation matrix.

\section{ACKNOWLEDGEMENTS}

The authors are grateful to the anonymous reviewers for their useful comments.

\section{FUNDING}

The biological data used in this article are part of a research project funded by the Société d'Accélération du Transfert de Technologies (SATT) Conectus Alsace (Dermapol project).

Dr. Jean Rehbinder was partly financed by the Agence Nationale de la Recherche (ANR), project DigitMC-PB, number ANR-18-CE19-0007-02.

\section{DISCLOSURES}

The authors declare no conflicts of interest.

\section{REFERENCES}

1. N. Ghosh and A. Vitkin, "Tissue polarimetry: concepts, challenges, applications, and outlook," J. Biomed. Opt. 16, 110801 (2011).

2. G. Anna, F. Goudail, and D. Dolfi, "Optimal discrimination of multiple regions with an active polarimetric imager," Opt. Express 19, 2536725378 (2011).

3. G. Anna, F. Goudail, and D. Dolfi, "Polarimetric target detection in the presence of spatially fluctuating Mueller matrices," Opt. Lett. 36, 4590-4592 (2011).

4. I. Vaughn, B. Hoover, and J. S. Tyo, "Classification using active polarimetry," in Polarization: Measurement, Analysis, and Remote Sensing $X$, vol. 8364 of Proceedings of SPIE (2012), p. 83640S.

5. J. Rehbinder, H. Haddad, S. Deby, B. Teig, A. Nazac, T. Novikova, A. Pierangelo, and F. Moreau, "Ex vivo Mueller polarimetric imaging of the uterine cervix: a first statistical evaluation," J. Biomed. Opt. 21 (2016).

6. J. Vizet, J. Rehbinder, S. Deby, S. Roussel, A. Nazac, R. Soufan, C. Genestie, C. Haie-Meder, H. Fernandez, F. Moreau, and A. Pierangelo, "In vivo imaging of uterine cervix with a Mueller polarimetric colposcope," Sci. Reports 7, 2471 (2017).

7. V. Tuchin, "Polarized light interaction with tissues," J. Biomed. Opt. 21, 071114 (2016).

8. S. Lu and R. Chipman, "Interpretation of Mueller matrices based on polar decomposition," J. Opt. Soc. Am. A 13, 1106-1113 (1996).

9. J. Gil, "Polarimetric characterization of light and media," Eur. Phys. J. Appl. Phys. 40, 1-47 (2007).

10. J. Gil, "Review on Mueller matrix algebra for the analysis of polarimetric measurements," J. Appl. Remote. Sens. 8, 081599 (2014).

11. J. Morio and F. Goudail, "Influence of the order of diattenuator, retarder, and polarizer in polar decomposition of Mueller matrices," Opt. Lett. 29, 2234-2236 (2004).

12. R. Ossikovski, A. De Martino, and S. Guyot, "Forward and reverse product decompositions of depolarizing Mueller matrices," Opt. Lett. 32, 689-691 (2007).
13. M. Anastasiadou, S. Hatit, R. Ossikovski, S. Guyot, and A. De Martino, "Experimental validation of the reverse polar decomposition of depolarizing Mueller matrices," J. Eur. Opt. Soc. - Rapid Publ. 2, 07018 (2007).

14. S. Alali and A. Vitkin, "Polarized light imaging in biomedicine: emerging Mueller matrix methodologies for bulk tissue assessment," Journal of Biomedical Optics. 20, 061104 (2015).

15. C. Heinrich, J. Rehbinder, A. Nazac, B. Teig, A. Pierangelo, and J. Zallat, "Mueller polarimetric imaging of biological tissues: classification in a decision-theoretic framework," J. Opt. Soc. Am. A 35, 2046-2057 (2018).

16. R. Ossikovski and J. Vizet, "Polar decompositions of negativedeterminant Mueller matrices featuring nondiagonal depolarizers," Appl. Opt. 56, 8446-8451 (2017).

17. S. Morgan and M. Ridgway, "Polarization properties of light backscattered from a two layer scattering medium," Opt. Express 7, 395-402 (2000).

18. Z. Xing, "On the deterministic and non-deterministic Mueller matrix," J. Mod. Opt. 39, 461-484 (1992).

19. G. Taubin, "3D rotations," IEEE Comput. Graph. Appl. 31, 84-89 (2011).

20. R. Horn and I. Olkin, "When does $A^{*} A=B^{*} B$ and why does one want to know?" The Am. Math. Mon. 103, 470-482 (1996).

21. R. Horn and C. Johnson, Matrix analysis (Cambridge University Press, 1985 , ch. 2, sec. 5, p. 100).

22. S. Altmann, Rotations, quaternions, and double groups (Clarendon Press, Oxford, 1986, ch. 9).

23. R. Ossikovski, M. Anastasiadou, and A. De Martino, "Product decompositions of depolarizing Mueller matrices with negative determinants," Opt. Commun. 281, 2406-2410 (2008).

24. B. Varin, J. Rehbinder, J. Dellinger, C. Heinrich, J. Schmidt, C. Spenlé, D. Bagnard, and J. Zallat, "Tumor growth monitoring using polarized light," in Novel Biophotonics Techniques and Applications V, vol. 11075 of European Conference on Biomedical Optics, SPIE Proceedings (2019).

25. J. Gil, R. Ossikovski, and I. San José, "Singular Mueller matrices," J. Opt. Soc. Am. A 33, 600-609 (2016).

26. R. Horn and C. Johnson, Topics in matrix analysis (Cambridge University Press, 1991, ch. 3, sec. 1, p. 147).

27. H. Noble and R. Chipman, "Mueller matrix roots algorithm and computational considerations," Optics Express 20, 17-31 (2012).

28. M. Karnoukian, S. Faisan, C. Heinrich, A. Lallement, and J. Zallat, "Segmentation of Mueller matrix images under non-uniform illumination," Opt. Express 23, 18218-18235 (2015).

29. R. Barakat, "Conditions for the physical realizability of polarization matrices characterizing passive systems," J. Mod. Opt. 34, 1535-1544 (1987). 